\title{
Objective Assessment of Covid-19 Severity Affecting the Vocal and Respiratory System Using a Wearable, Autonomous Sound Collar
}

\author{
D. Ishac, ${ }^{1}$ S. Matta, ${ }^{1}$ S. Bin, ${ }^{2}$ H. Aziz, ${ }^{3}$ E. Karam, ${ }^{1}$ A. Abche, ${ }^{1}$ and G. Nassar (i) ${ }^{4}$ \\ ${ }^{1}$ Electrical Engineering Department, University of Balamand (UOB), Balamand, Lebanon; ${ }^{2}$ College of Physics, University of \\ Qingdao, Qingdao, China; ${ }^{3}$ Department of Pulmonary Pathology, Sahlgrenska University Hospital, Göteborg, Sweden; and \\ ${ }^{4}$ IEMN - CNRS UMR 8520-INSA (HdF)-Lille academic, Lille, France
}

(Received 4 March 2021; accepted 11 October 2021; published online 5 November 2021)

Associate Editor Michael R. King oversaw the review of this article.

\begin{abstract}
Introduction - Since the outbreak began in January 2020, Covid-19 has affected more than 161 million people worldwide and resulted in about 3.3 million deaths. Despite efforts to detect human infection with the virus as early as possible, the confirmatory test still requires the analysis of sputum or blood with estimated results available within approximately 30 minutes; this may potentially be followed by clinical referral if the patient shows signs of aggravated pneumonia. This work aims to implement a soft collar as a sound device dedicated to the objective evaluation of the pathophysiological state resulting from dysphonia of laryngeal origin or respiratory failure of inflammatory origin, in particular caused by Covid-19.

Methods - In this study, we exploit the vibrations of waves generated by the vocal and respiratory system of 30 people. A biocompatible acoustic sensor embedded in a soft collar around the neck collects these waves. The collar is also equipped with thermal sensors and a cross-data analysis module in both the temporal and frequency domains (STFT). The optimal coupling conditions and the electrical and dimensional characteristics of the sensors were defined based on a mathematical approach using a matrix formalism.

Results - The characteristics of the signals in the time domain combined with the quantities obtained from the STFT offer multidimensional information and a decision support tool for determining a pathophysiological state representative of the symptoms explored. The device, tested on 30 people, was able to differentiate patients with mild symptoms from those who had developed acute signs of respiratory failure on a severity scale of 1 to 10 .

Conclusion - With the health constraints imposed by the effects of Covid-19, the heavy organization to be implemented resulting from the flow of diagnostics, tests and clinical management, it was urgent to develop innovative and safe biomedical technologies. This passive listening technique will contribute to the non-invasive assessment and dynamic observation of lesions. Moreover, it merits further examina-
\end{abstract}

Address correspondence to G. Nassar, IEMN - CNRS UMR 8520-INSA (HdF)-Lille academic, Lille, France. Electronic mail: gnassar@uphf.fr tion to provide support for medical operators to improve clinical management.

Keywords-Autonomous sound systems, Mechanical variables measurement, Electromechanical sensors, Sound signal processing, Covid-19.

\section{INTRODUCTION}

Nowadays, smart devices play an important role in various aspects of our daily lives. Some of these devices will even think for us. In all technological sectors, researchers are focusing on the design or optimization of operations to generate a new model of interaction between the different actors of society involved in the areas of health, comfort, and safety. ${ }^{21}$ Far from replacing the role of professionals, onboard electronic systems strengthen the links between scientists, patients, and caregivers. This practice of "continuous monitoring" reassures individuals and allows them to play a more proactive role in their healthcare.

Since the outbreak began in January 2020, Covid-19 has affected more than 161 million people worldwide and resulted in about 3.3 million deaths. ${ }^{12}$ Despite efforts to detect human infection with the virus as early as possible, the confirmatory test still requires the analysis of sputum or blood with estimated results available within approximately 30 minutes; this may potentially be followed by clinical referral if the patient shows signs of aggravated pneumonia. This pandemic has mobilized a large number of research centres that have studied the dynamics and dissected the structure and genome of the virus to limit its consequences in terms of its potential for contamination and mutation. 
Most research currently focuses on methods of biological screening, such as the Reverse Transcriptase (RT) Polymerase Chain Reaction (PCR) ${ }^{2}$ test or physical screening using $\mathrm{x}$-rays combined with deep learning, for example, which aim to provide a rapid, fully automatic diagnosis. ${ }^{1,8,18}$ Early detection of the disease would enable the implementation of the proper care required by patients with Covid-19.

Diseases such as the H1N1 influenza A virus, and recently Covid-19, affect the vocal and respiratory system leading to an inability of the lungs to transfer enough oxygen to the body. Therefore, it has become essential to implement systems of prediction, diagnosis, and "real-time" analysis to cope with health crises.

Recent efforts have shown promise in terms of improving detection in several medical fields and are based primarily on three categories: (1) mathematical approaches to estimate uncertainty in Deep Learning for the detection of infectious viruses, in particular coronavirus $^{4,5,13}$; (2) analytical methods to improve the management of the pandemic and its effects while taking into account peculiarities and genetic sensitivity during and after infection ${ }^{9,17,33,46}$; and (3) radiological classification focusing more particularly on deep learning models. ${ }^{14,30,36,44}$

This work aims to implement a sound device for the objective evaluation of the pathophysiological state resulting from dysphonia of laryngeal origin or respiratory failure of inflammatory origin, in particular caused by Covid-19. The resulting platform comprises modules for analyzing physiological data provided by biocompatible acoustic and thermal sensors. The latter focus on passive listening of the vocal and respiratory system to offer health professionals multidimensional information and a decision support tool for determining a pathophysiological state representative of the symptoms explored. The idea is to ensure real-time, remote diagnosis (rural, isolated areas, etc.), thus optimizing time, treatment, and referral to the right department. Ultimately, the aim is to establish standardization charts resulting from a wide "clinical" validation plan representing a broad range of the population.

\section{BACKGROUND}

In the vocal acoustic domain, the voice has been the subject of several studies; the majority are oriented towards speech recognition to use the voice components as a security key or an actuator. In the medical field, the literature shows some work exploiting the vibrational components for the diagnosis of acute lung injury using devices in ultrasound scan mode. ${ }^{6,10,29}$ In this way, the use of ultrasound has been proposed for the detection and management of pneumonia in paediatric populations ${ }^{3,40}$ and adults. ${ }^{29}$ Recently, in the context of the Covid-19 pandemic, research has been undertaken on the detection of serum affecting the pulmonary alveoli resulting from novel pneumonia or Acute Respiratory Distress Syndrome (ARDS), for instance Refs. 11, 16, 37, 38, 41, and 42 .

A few studies have focused on the use of the vibratory mechanism of laryngeal tissues to define some pathological cases. Among these studies, Guen ${ }^{24}$ focused on vibratory models of the vocal cords using video laryngoscopy providing images reflecting their vibratory motions. In 2018, Bowen ${ }^{7}$ carried out work on five subjects to estimate the vibratory waves produced by the mucous tissues based on the electroglottography principle. Sanaz ${ }^{43}$ examined the effect of Vibro Tactile Stimulation on voice/speech quality for therapeutic purposes and showed that laryngeal VTS modulates neural synchronization.

Advances in both "soft" and "hard" technologies have enabled the development of applications based on the concept of Artificial Intelligence through the combination of several functionalities. In this context, Turkman $^{48}$ focused on an image segmentation approach for the assessment of laryngeal disorders. In the same context, research by Fehling ${ }^{19}$ and Hyun ${ }^{25}$ explored the glottal area with high-speed laryngeal imaging (HSV) using a deep convolutional neural network to detect certain failures in the vibratory mechanism. Analysis of this recent literature shows that the use of artificial intelligence to assess the failure of the phonic system is more oriented towards the sequential sampling of the video images; this requires the positioning of the device inside the larynx, facing the vocal cords (epiglottis zone).

Recently, Matta ${ }^{32}$ published a study on an acoustic technique analysing the vibratory components of the vocal system for the diagnosis and early detection of a potential cancerous state. The results of this technique highlighted the dependence of the vibratory characteristics of the collar on the pathophysiological state of the patient. The current work is an extension of the passive sound device used by Matta ${ }^{32}$ and Ishac ${ }^{26,27}$ for the early detection of abnormalities of the laryngeal apparatus. This extension concerns additional hardware and algorithmic modules ensuring the combination of physiological data flows for the objective analysis of the severity of the effects of the Covid-19 virus. 


\section{MATERIAL AND METHODS}

\section{Multilayer Tissue-Wave Interactions}

The vocal and respiratory system generates waves causing a multilayer tissue medium to vibrate. Vibratory sensors appropriately distributed around the patient's neck collect these waves. However, the spatiotemporal parameters of the system need to be adjusted for preventive and/or diagnostic purposes to improve system performance, as well as the evaluation of wave-biological tissue interactions. Thus, the optimal coupling conditions and the electrical and dimensional characteristics of the sensors were defined based on a mathematical approach using a matrix formalism. Matta $^{31}$ showed that the displacement/stress vectors are spatiotemporally evaluated in the propagating tissues in accordance with their physical properties (Fig. 1). Consequently, the design and operating conditions of the measurement system were adjusted to reflect the conditions under consideration, i.e., optimization of the coupling coefficient at the interface and the selection criteria of the sensors.

\section{Physical Device (Collar)}

As mentioned above, the collar that is the subject of this study is an extension of the device used in the work of Matta. ${ }^{32}$ Flexible bio-compatible poly-vinylidenefluoride polymer (PVDF) sensors were used to ensure a fairly wide frequency bandwidth covering both the respiratory (pulmonary) and phonatory mechanism. These sensors made from electro-active nanowires resulting from electrospinning technology 28,49 were added and used in place of the concentric ring PZT receptors (PZ26 Ferroperm Sct.) produced by laser engraving (Fig. 2).

\section{Acquisition System}

The device has been adapted for the diagnosis of symptoms linked to the effects of Covid-19. Its acquisition system comprises primarily the following modules: an electroactive sound module, a thermoelectric module, and an electromechanical module.

\section{Electroactive Sound Module}

PVDF polymers have been favoured as electroactive materials for their biodegradable organic properties and the appreciable piezoelectric constant $\mathrm{d} 33$ of about $20 \mathrm{pCN}-1 .{ }^{20,34,45}$ To ensure the optimal performance of their electrical and mechanical characteristics, the nanowire-based PVDF sensors ${ }^{47}$ embedded in the collar were improved by doping with Barium Titanite (BaTiO3) and graphene fibres $(\mathrm{GNr})$.
The study and characterization of these nanowire sensors (PVDF + doping components) showed that the electromechanical performance was optimal with the following proportions of doping components: $3 \%$ BaTiO3 and 0.25\% GNr (Supplementary Figure 1).

The tissue sensors embedded in the collar were calibrated in transmission mode to quantify their stability and sensitivity in reference media: water and agar during the sol-gel transition. The various curves in Supplementary Figure 2 shows the stability of the acoustic characteristics (velocity, wave time of flight, and attenuation) in water at a controlled temperature of $23{ }^{\circ} \mathrm{C}+/-0.1{ }^{\circ} \mathrm{C}$, as well as the sensitivity for monitoring evolving complex phenomena (viscoelastic behaviour in phase transition). Good reproducibility was observed with a dispersion of less than $5 \%$ for the four follow-up measurements carried out under the same operating conditions.

\section{Thermoelectric Module}

The collar is equipped on each side of the neck with two thermal sensors measuring $5 \mathrm{~mm} \times 5 \mathrm{~mm} \times 1 \mathrm{~mm}$, positioned at $70^{\circ}$ and $-70^{\circ}$ with respect to the frontal axis. The role of these two sensors is to provide an estimate of the average body temperature when the measurements are acquired.

The operating principle involves producing thermoelectric energy based on the Seebeck effect. A bismuth telluride (Bi2Te3) semiconductor material was chosen for the quality of its thermoelectric conversion over a bandwidth ranging from -60 to $+125^{\circ} \mathrm{C} .{ }^{23}$

\section{Electrical Module}

The electrical module (Fig. 3), an autonomous system integrating scheduling tasks and a transfer functionality, converts vibratory mechanical energy ${ }^{15}$ into electrical energy to satisfy the energy needs of the hard and soft modules of the collar.

The module was produced using a System-on-Chip (SoC) from the Texas Instruments MSP430 family. The MSP430F2012 model was chosen for its small size and minimal hardware resources (with a USB port). This SoC includes a central processing unit (CPU), a digitally controlled oscillator (DCO), a task scheduler (IT), an accumulator (3-volt DC), a coded sensor generator (PWMG), a power management unit (PMU), and an output amplifier (OB). A power management unit was added to this modular architecture to allow greater autonomy. The average consumption of the collar is $0.026 \mathrm{~mA}$ when the power unit is on standby (until the vibration of the vocal cords activates the system). Associating a user interface with the collar functionalities has turned this concept into a medical platform capable of evolving and adapting to an e- 


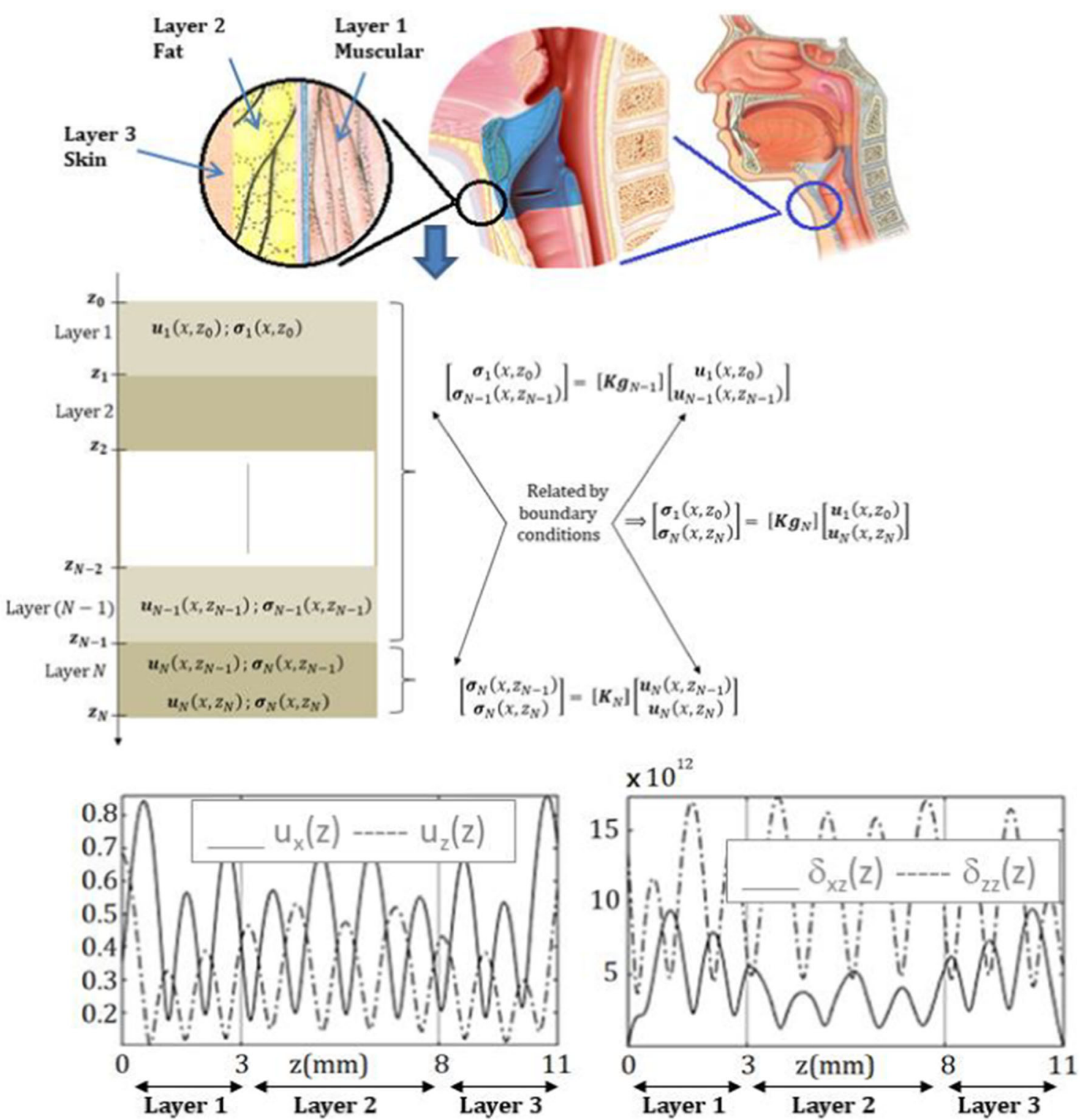

FIGURE 1. Diagrams of the displacement-stress vector components showing the variation in amplitude of the incident sound wave (vibrational) through tissue layers constituting the phonic apparatus zone. A study seen under a multilayer formalism. ${ }^{31}$.

Health environment under the TCP/IP ${ }^{32,35}$ protocol and/or via a specific smartphone application for remote physiological monitoring 22 (Fig. 4).

\section{ANALYTICAL APPROACH}

Covid-19 is characterized by severe pneumonia and/ or acute respiratory distress syndrome in approximately $20 \%$ of infected patients. The urgency of the health situation has led to the prioritization of the search for indicators reflecting the severity of infection in affected people to orient them effectively, optimize rapid management, and guide clinical intervention. It is in this context that the approach developed con- verges towards the establishment of a multiplex telemonitoring platform to improve surveillance efforts. Additional tests such as protein serology and/or nucleic acid tests can be combined if necessary. Therefore, to quantify the severity of the syndrome in affected people, an acoustic and thermal signature was established for each suspected case. The combination of this information allows a Decision Support System to generate a report on the severity and evolution of each person's condition. In fact, the vocal and respiratory characteristics could reveal possible physiological symptoms related to the modifications of the spectro-temporal morphology of the signals, thus allowing pathological classification. The diagram in 

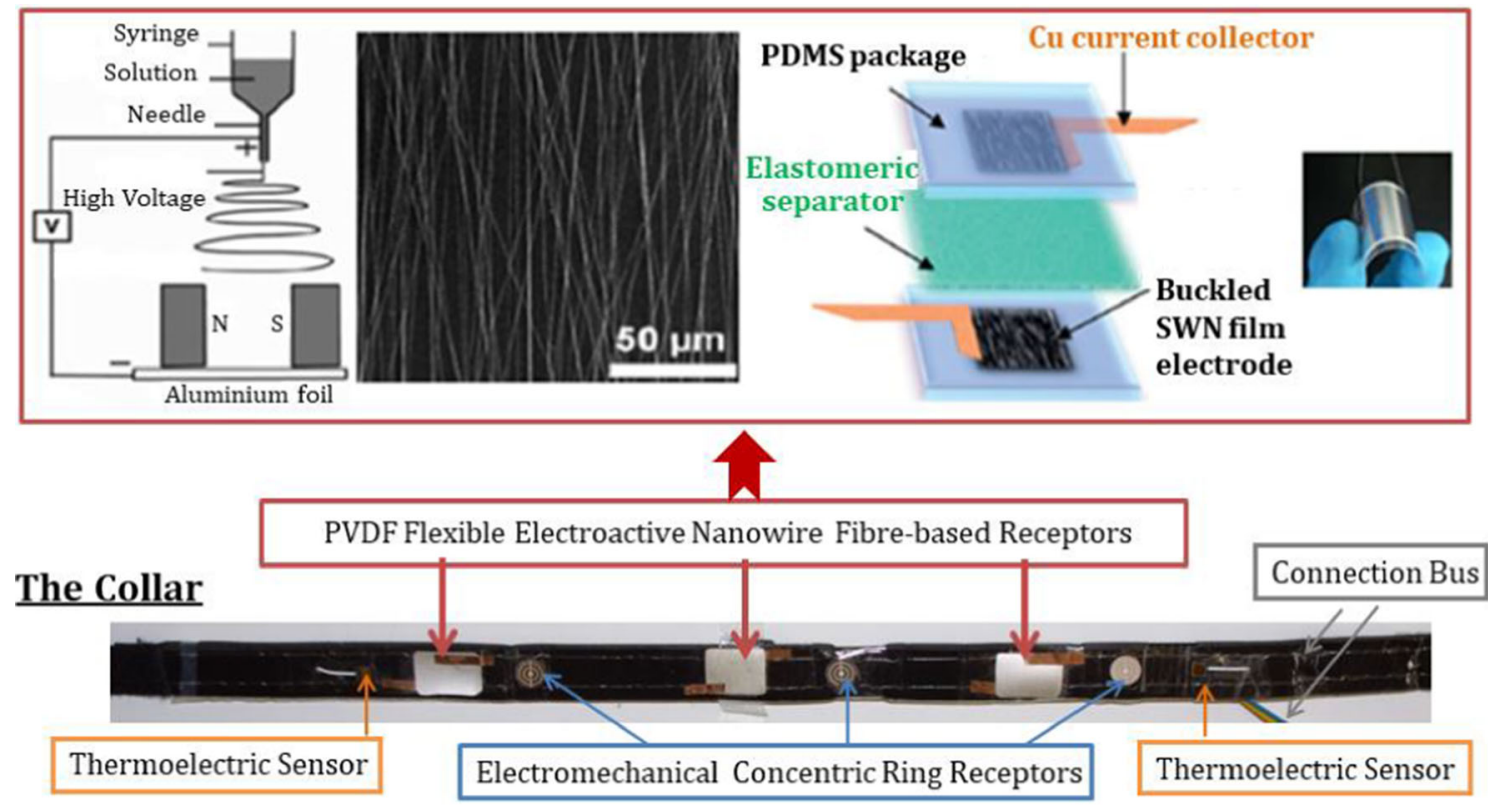

FIGURE 2. Schematic diagram of the different components of the collar. The electroactive nanowire-based vibratory sensors were developed using electrospinning technology with PVDF. ${ }^{47}$ These flexible thin film sensors were integrated into the inner part of the collar in contact with the skin. This device also has two thermal sensors whose role is to ensure an average measurement of the body temperature. Each item is connected to a central electrical unit.

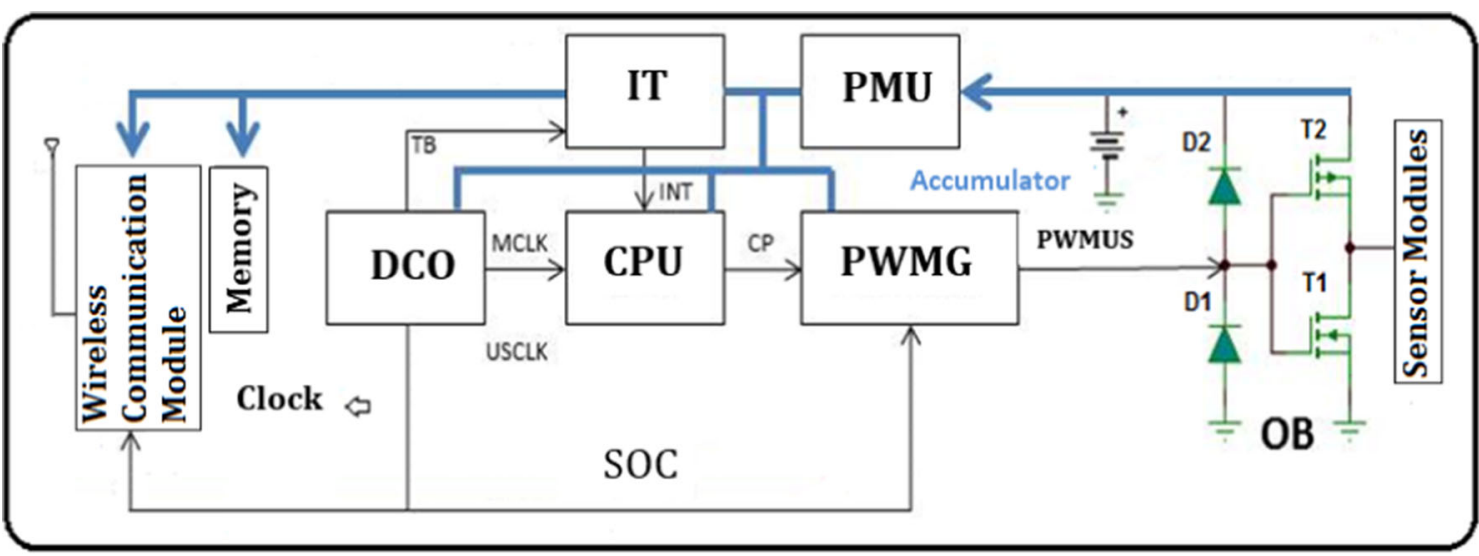

FIGURE 3. Architecture of the real-time electrical unit comprising the central scheduling tasks, sensor management, and wireless communication module.

Fig. 5 illustrates the overall approach implemented for acquisition, analysis, and evaluation.

\section{Feature extraction (Supplementary Table)}

Relative Time Dispersion (RTD)

The $R T D$ is the deviation of the temporal agitations between the first cycles (periods), cycle by cycle, of each signal (the first 5 cycles in our study), thus reflecting any cyclical instability in the time signal parameters. A relatively large fluctuation is strongly linked to a disorder of the vibratory (phonic) mechanism of the subject. This quantification of the very short-term vibrational disturbance of the signal is given by the following expression:

$$
R T D=2 \delta=\frac{\frac{1}{K-1} \sum_{i=1}^{K-1}\left|T^{(i)}-T^{(i+1)}\right|}{\frac{1}{K} \sum_{i=1}^{K} T^{(i)}}
$$

where the subscript (i) refers to the $i$ th cycle and $K$ is the number of cycles.

\section{Correlation}

Correlation (Cor) is an approach that highlights the similarity between two different time signals $\mathrm{x}(\mathrm{t})$ and $y(t)$ and is given by the following expression: 


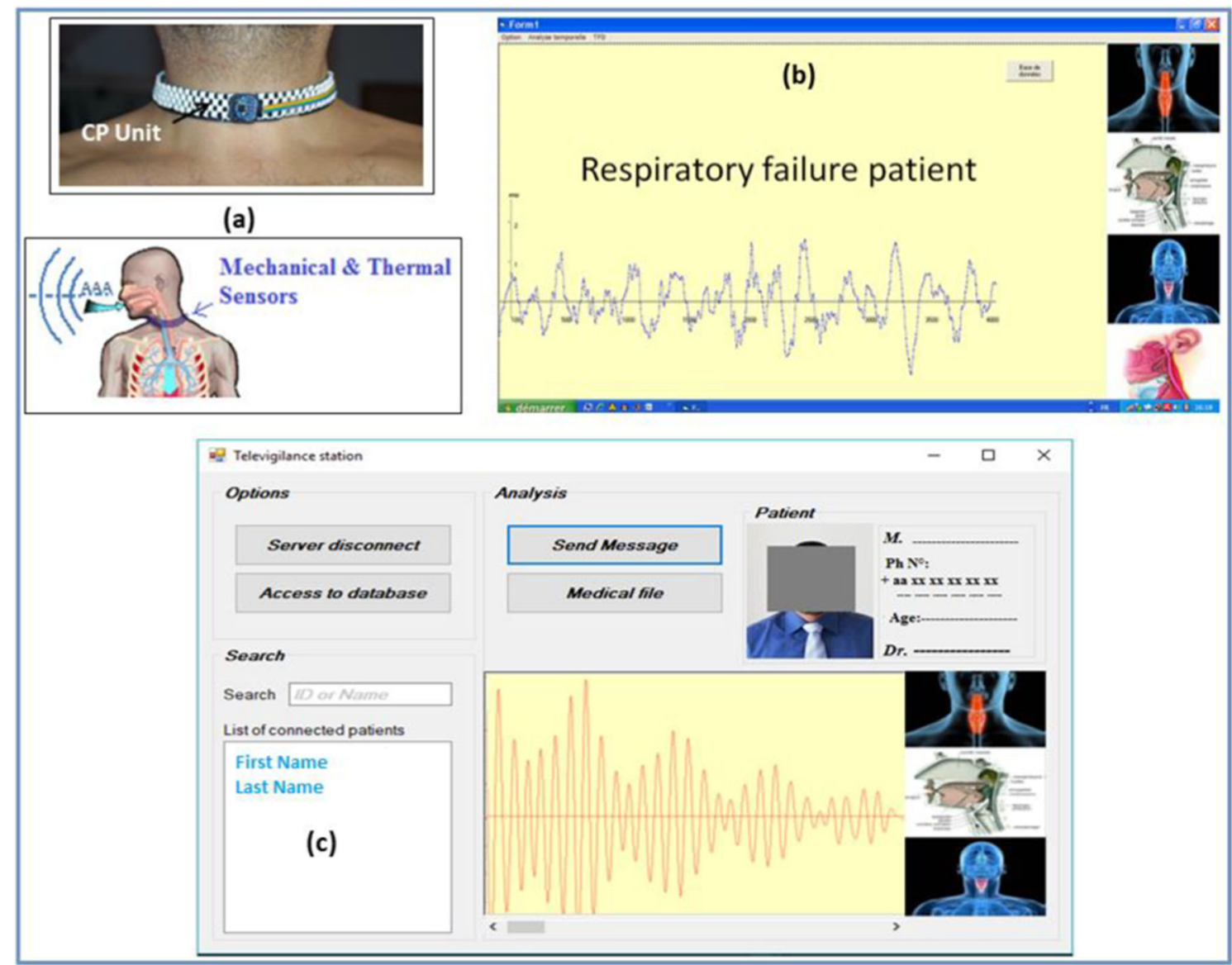

FIGURE 4. (a) Placement of the collar and excitation modalities by respiration and/or pronunciation of the vowel /a/; (b) User interface display on a local USB terminal and/or (c) through a smartphone application.

$$
W_{x y}(\tau)=E(x(t) \cdot y(t-\tau))
$$

If we consider $x(t)$ as the signal characterizing the physiological state of a subject at D0 day, $y(t)$ is the signal acquired at D0 $+\mathrm{Di}(i=1 \ldots 15)$. Depending on the sampling stage, the effect of Covid-19 on an affected subject modifies the morphology of the original signal $(x(t))$, which can be measured using the Correlation Coefficient. Considered a decisive indicator, its evolution is explained by the modification, extinction, or appearance of certain characteristic components of the starting signal.

\section{Frequency Components}

Similar to temporal analysis, the frequency content is also rich in information concerning the evolution of the pathophysiological state of the patients. The vocal and respiratory signals collected can be considered as non-stationary signals and, therefore, have to be analysed using a time-frequency approach to extract the existing frequencies and their respective time of occurrence. Consequently, the Short-Time Fourier
Transform (STFT) was performed in conjunction with a Gaussian window to determine the spectrogram (Fig. 6) and identify the frequency components (position and peaks).

\section{Indices}

After collecting then processing the time signal using STFT, the peaks were localized by thresholding and the corresponding frequencies were extracted from the spectrum. The average value of each index $\left(F_{\mathrm{I}}\right)$ was identified by the position of its maximum on the frequency axis. Its content was estimated by its full width at half maximum (FWHM) and it is defined as:

$$
F_{I Q}=\sqrt{\frac{\sum_{k=1}^{N}\left(\left(F_{I}^{(k+N / 2)}-F_{I}^{(k-N / 2)}\right)\right)^{2}}{N}}
$$

where $N$ is the number of samples in the frequency band (window), $F_{\mathrm{I}}$ is the average value defined as $F_{I}=$ $\frac{1}{N} \sum_{k=1}^{N} F^{(k)} . F_{\mathrm{I}}$ is the $k$ th frequency in the frequency band (window). 


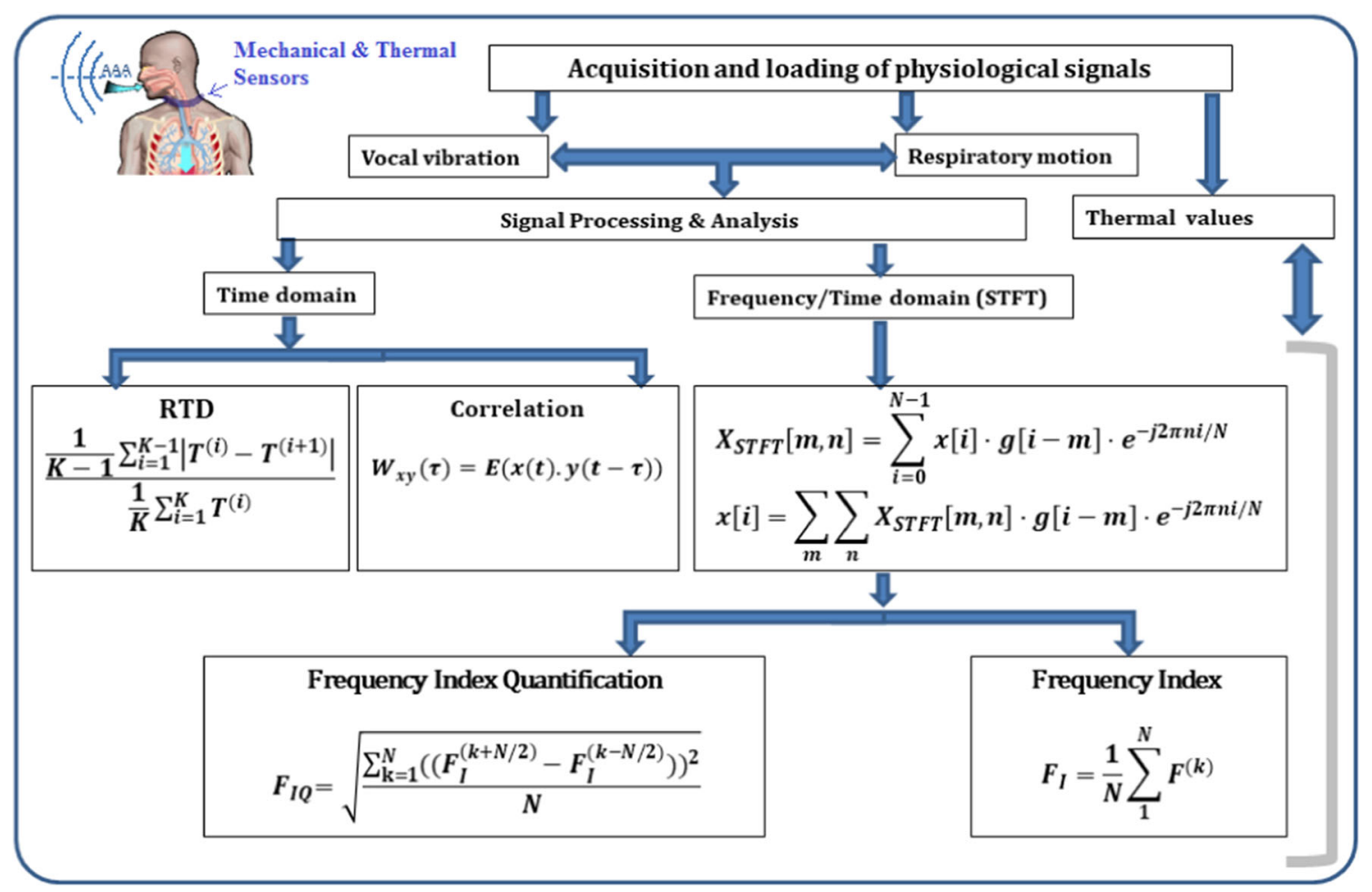

FIGURE 5. Block diagram showing the extraction of the features from the signals collected using time-based and frequencybased approaches. The temporal quantities are coupled with the extraction of the frequency characteristics based on the STFT and the body temperature. Where $F_{1}$ is the frequency index, $F^{(\mathrm{k})}$ is the ith frequency in the frequency band (window), $F_{1 Q}$ is the Frequency dispersion, $N$ is the number of samples in the frequency band (window), RTD is the relative time dispersion, $W_{x y}(\tau)$ is the correlation function.

\section{CLASSIFICATION}

The time and frequency characteristics, as well as the evolution of the patient's fever $\left(T^{\circ} \mathrm{C}\right)$, enable the patient's diagnosis, an objective evaluation, and a decision to be made by the expert module. The patient is classified according to the clinical severity of his/her condition (by category and by group) by evaluating the expression of Severity (S) normalized with respect to a normal healthy subject (Reference):

$S=f($ weighted $Q) \times \% \operatorname{Ref} Q$

$=\sum \Delta\left(x_{k}, x_{R e f}\right)$ where $D$ is a subtraction operator

where $x_{k}$ are the features $\left(2 \delta,(1 / n), \Delta F, F_{I Q}\right.$, (Cor Coef), $T^{\circ} \mathrm{C}$ ).

This approach leads to a new procedure that exploits the physiological quantities from structural vibratory mechanisms. To identify and cluster each category, an intra-group variance study of the basic characteristic quantities (fever and frequency components) was evaluated.

The physiological functionalities constituting the indicator " $Q$ " were identified according to their strong dependence on the vibratory characteristics resulting from the effects of Covid-19. Indeed, the temporal disturbance $2 \delta$ is reflected in the frequency domain by a disorder that, in severe cases, results in a shift and/or extinction of the significant frequency components (indices). Consequently, " $n$ " is defined as the number of these components.

$\Delta \boldsymbol{F}$ and $\boldsymbol{F}_{\boldsymbol{I Q}}$ are defined as the difference between the frequency indices of the diagrams at different acquisition times and the Frequency Width at Half-Maximum (FWHM) of each index. They are strongly affected by the disturbance of the phonic and respiratory mechanism. As the severity of the effects increases, $\Delta \boldsymbol{F}$ migrates towards a lower frequency and the FWHM of $\boldsymbol{F}_{I \boldsymbol{O}}$ increases.

Consequently, " $Q$ " is a multidimensional indicator that amplifies decisive signs. When associated with a relatively high fever and a correlation coefficient that evolves (decreases) between two consecutive measurements on an infected subject, and after normalization with respect to the reference state (subjects with stable features), it provides a decision threshold on the criticality of the situation.

The starting value of approximately 0.65 for the correlation coefficient results from the extra physiological variation in healthy subjects (average autocorrelation of 0.95 for the same subject). 


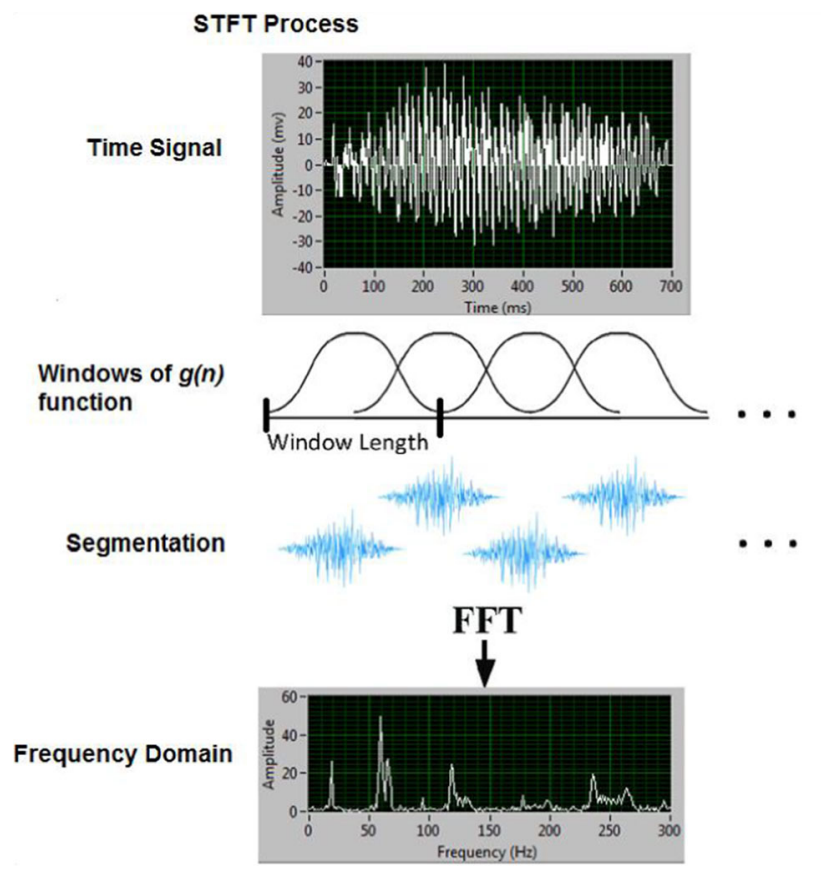

FIGURE 6. Schematic illustration showing the principle of the STFT function for monitoring the temporal variation harmonics of the signals. The principle consists in segmenting the signal with a sliding window of Gaussian form (function $g(n)$ ). The depth and sliding step are optimized according to the frequency content sought by applying the FT to each window separately. This reveals the Fourier spectrum of each shorter segment with a global display cascading the frequency components found.

\section{APPLICATION AND RESULTS}

A study was conducted on 30 subjects aged 53 to 60 years with an equal number of males (M) and females (F). Patient follow-up was only authorized during the first 2 weeks (limiting our intervention outside the hospital environment) with three measurements per week and per person (3rd day, 5th day, etc.). The subjects were divided into 2 categories: unaffected and affected (positive PCR test). The latter was split into two subgroups depending on whether the cases were asymptomatic or severe.

1. Category 1: Healthy subjects (5)

2. Category 2: Affected subjects (25)

a. Subgroup 1: 5 subjects who had more or less noticeable mild flu symptoms and subsequently declared as asymptomatic.

b. Subgroup 2: 20 subjects who had a fever, a cough, and more or less acute respiratory distress.

The thermal, vibratory, and respiratory signals were acquired from each person while uttering the vowel /a/ followed by a cycle of deep breathing to excite a wide frequency range from the laryngeal system (voice and respiration). For each person, an average of three signals per sample was taken into account to reduce dispersion associated with the individual and the positioning of the collar.

The study was limited to 30 people due to the strict health context, thus generating limitations regarding the number of subjects and the frequency of applications. The number of healthy and asymptomatic subjects (without significant symptoms) was thereby limited to 5 per group so more attention could be devoted to those who had developed advanced symptoms very quickly. Nevertheless, we were able to optimize our intervention method according to the clinical instructions of the health professionals while aiming for the initial validation of our concept in terms of screening and sensitivity. The affected subjects were followed every 2 days over the 2 weeks in conditions that still allowed the positioning of the collar and the acquisition of the signals. However, this was no longer possible once the patient's condition became worrying and required admission to the dedicated service.

\section{Physiological Signature: Acoustic and Thermoelectric (Fever)}

\section{Healthy Subject}

The subjects were monitored by attaching the collar around the neck. As mentioned earlier, an average of three measurements per subject were performed to reduce the dispersion (STD) to about 4\% (including gender).

Figure 7 shows a typical vocal vibration signal collected from a healthy subject when pronouncing the vowel /a/. This illustration shows the temporal signal with a duration of $700 \mathrm{~ms}$ and an amplitude varying between $-40 \mathrm{mV}$ and $+40 \mathrm{mV}$. It also depicts the frequency decomposition using STFT with a Gaussian sliding window of the function " $\mathrm{g}$ " with a depth of 100 $\mathrm{ms}$ and an increment of $10 \mathrm{~ms}$.

Table 1 shows the average values of the features at three representative stages: D0, D8, and D14 for healthy subjects. The stability of these values reflects their physiological state, which will be considered as the reference.

To quantify the difference between genders, Fig. 8 illustrates the localization of the frequency indices $\left(\mathrm{F}_{\mathrm{I}}^{(i)}, i=1 \ldots 4\right)$ of two healthy subjects (one male and one female). The features were extracted from the STFT spectrum. The plots show that the two genders (M \& F) have more or less the same frequency patterns but with a slight shift to the lower frequencies for the male subject. These results are consistent with the work carried out on voiceprints. ${ }^{39}$ However, the reference respiratory signals show that the variations between the genders were not significant. The signals were 


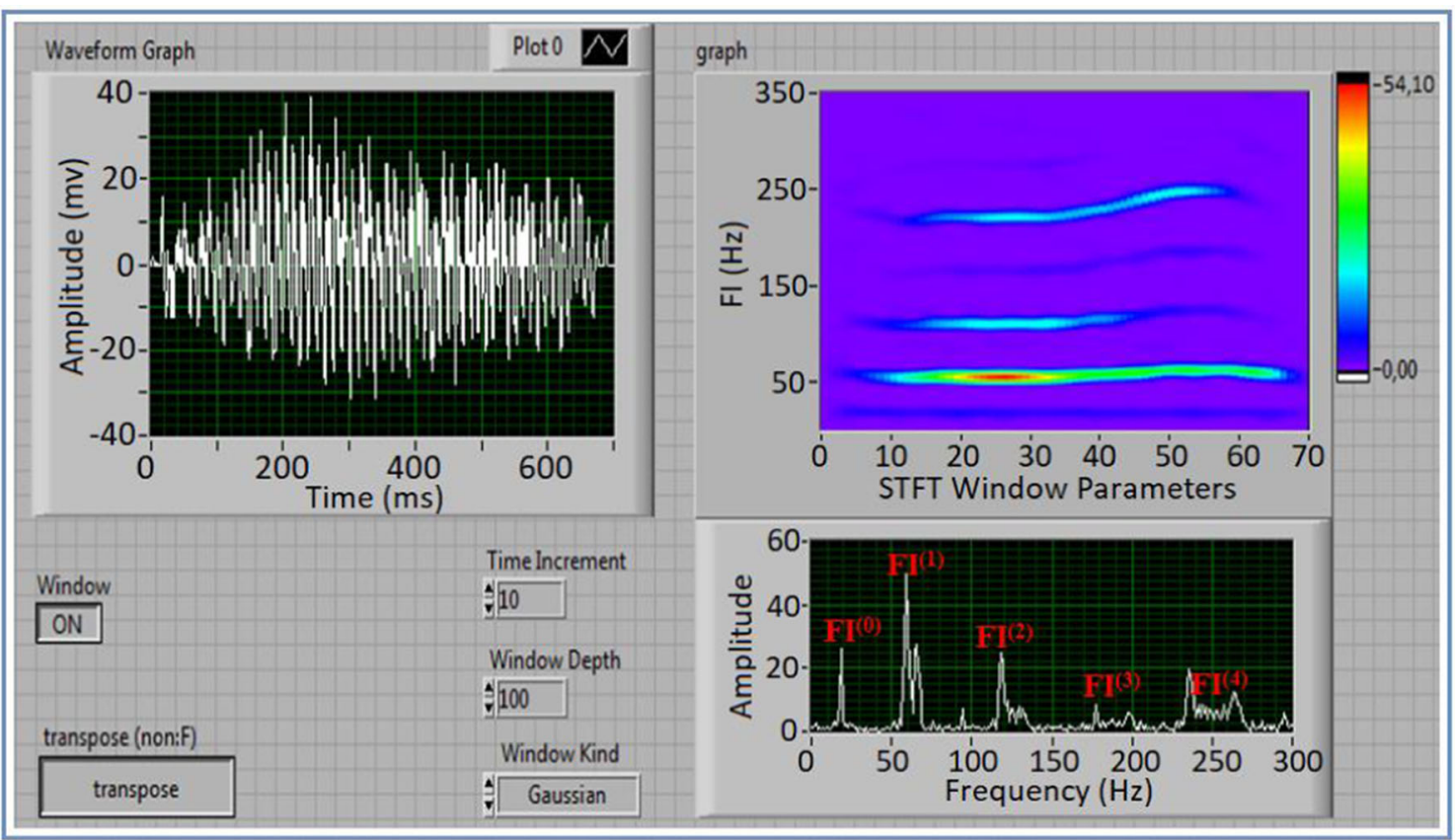

FIGURE 7. The (STFT) applied to the temporal phonic signal of a healthy subject (top left) with a Gaussian function $\mathrm{g}(\mathrm{n}) 100 \mathrm{~Hz}$ in depth and an increment of $10 \mathrm{~Hz}$ (bottom left) reveals the frequency content in the form of Indices (FI) represented both by the intensities (top right) of each component and a frequency spectrogram (bottom right).

TABLE 1. Average values of extracted features in healthy subjects.

\begin{tabular}{llccc}
\hline $\begin{array}{l}\text { Healthy } \\
\text { Subjects }\end{array}$ & $2 \delta(\mathrm{ms})$ & $F_{\mathrm{l}}^{(1)}(\mathrm{Hz})$ & $F_{\mathrm{IQ}}(\mathrm{Hz})$ & Fever $\left(T^{\circ} \mathrm{C}\right)$ \\
\hline \multicolumn{5}{c}{ Do (average cor coef between subjects of 0.69$)$} \\
Average & 1.51 & 60.59 & 7.60 & 36.6 \\
STD & 0.01 & 0.90 & 0.55 & 0.2 \\
& D8 (average cor coef between subjects of 0.66$)$ \\
Average & 1.52 & 60.03 & 7.60 & 36.61 \\
STD & 0.01 & 0.30 & 0.55 & 0.25 \\
& D14 (average cor coef between subjects 0.67$)$ \\
Average & 1.51 & 60.06 & 7.70 & 36.72 \\
STD & 0.02 & 0.11 & 0.53 & 0.22 \\
\hline \multicolumn{5}{r}{}
\end{tabular}

collected with an average body temperature of $36.7^{\circ} \mathrm{C}$ around the neck.

For the remainder of this application and the age group examined, we observed that the frequency variations (of the phonic apparatus) resulting from the effects of Covid-19 were much higher than the intergender $(\mathrm{M} / \mathrm{F})$ variations, which is why this parameter was not taken into account in our analysis.

\section{Subjects Tested Positive (PCR Test)}

\section{Asymptomatic}

Among the subjects participating in this study, five tested positive with an evolution observed (clinically and through data analysis) towards an asymptomatic

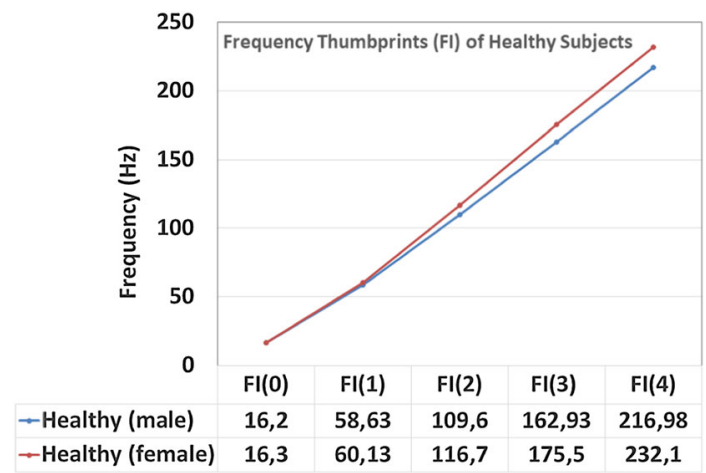

FIGURE 8. Typical graph showing the very close localization of the frequency indices of vibrating signals produced by the vocal apparatus of two healthy subjects of the same age but different gender. The curves show that the vocal thumbprint of a female is slightly higher (more acute) than that of a man.

state. However, despite the lack of severe symptoms, the analysis showed a slight modification in the frequency thumbprints measured or indices (Fig. 9a). This characteristic change, particularly in the highfrequency indices, correlated with an average body temperature of $37.2^{\circ} \mathrm{C}$, can be attributed to the physiological effects of the virus on the body.

Conversely, the curves in Figs. 9b-9d show the variation in the time and frequency components versus the evolution of the effects of Covid-19. The correlation coefficient is considered relative to a reference value (average of healthy subjects) and not its own value (auto-correlation of 0.92 ) in the category. This is 


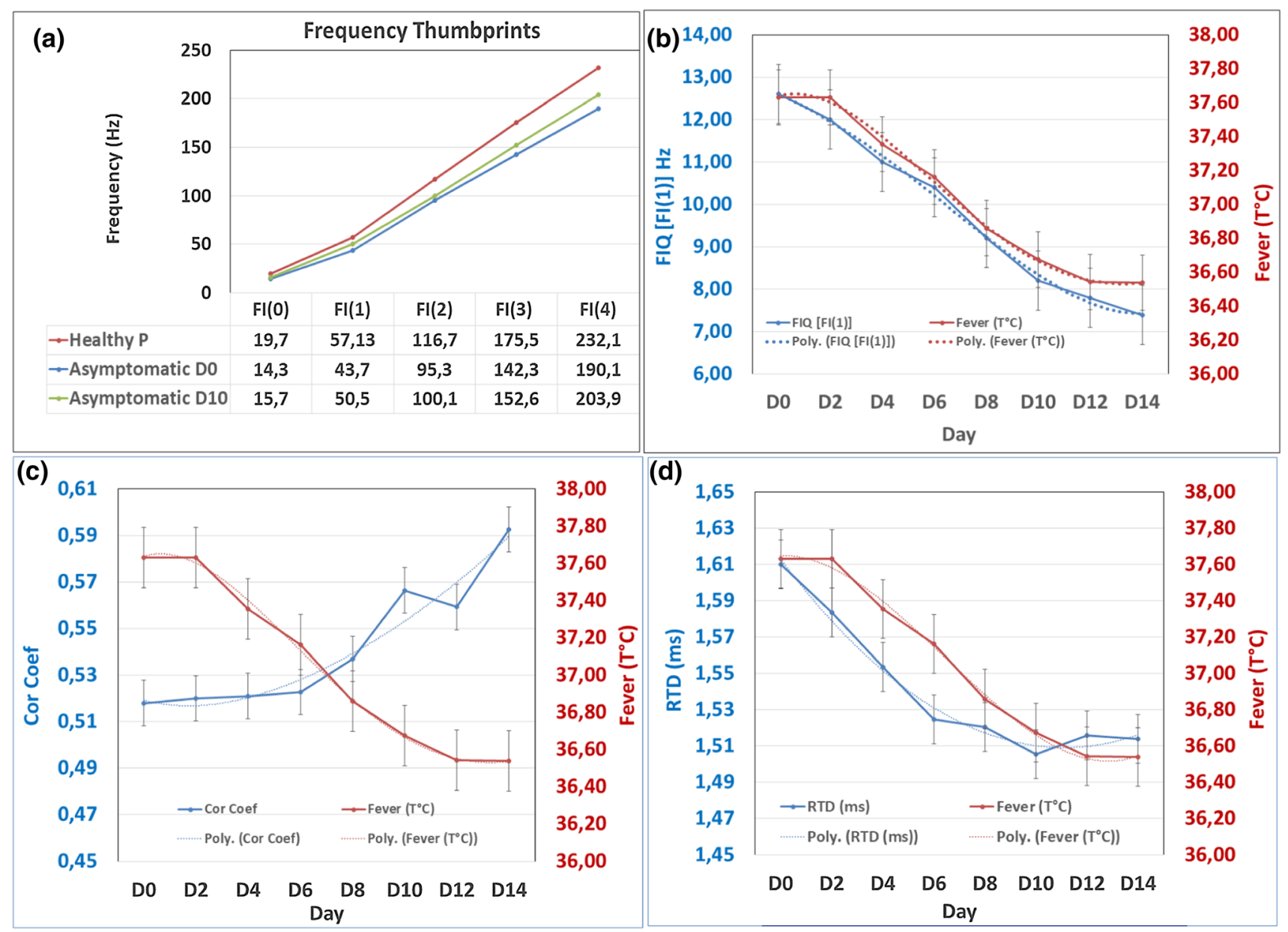

FIGURE 9. (a) $\mathrm{FI}^{(\mathrm{i})}$ frequency diagram of an asymptomatic subject (compared to a healthy one) over two different periods; D0 positive PCR test and 10 days later (D10). (b-d) Evolution (D0 to D14) of the physiological characteristics of the signals showing the tendency of the quantities towards a reference level (healthy subject).

why the starting value on the curves is of the order of 0.6 , thus reflecting the difference in the morphology of the signals between a healthy and an asymptomatic state. The tendency of the curve (coupled with other quantities) towards higher values reflects the successive disappearance of the effects of Covid-19.

Even if the respiratory assessment for this category did not show any significant changes, the vocal plot confirms the successive return (after 2 weeks) of the high-frequency components reflecting a pathological regression for the subject. This was confirmed by a negative PCR test performed 2 weeks after the initial positive test, indicating a strong correlation with the effects of the virus.

The data converged towards a stable state after the 8 th day. The initial temperature, which was around $37.6{ }^{\circ} \mathrm{C}$ (D0 PCR), asymptotically decreased to an average value of $37.02^{\circ} \mathrm{C}$ after 1 week, with a tendency to reach the average reference value of $36.6^{\circ} \mathrm{C}$. The other features observed also reflect a return to a normal physiological state. For this category, no sig- nificant variation in the correlation coefficient of the respiratory system was observed.

\section{Affected Subject (Isolation)}

Collar readings were performed every 2 days (over 15 days) on the patients with a positive Covid-19 PCR test (subgroup 2), i.e., 20 subjects exhibiting pronounced Covid-19 symptoms of varying severity. The measurements were combined with PCR tests to quantify the performance of the acoustic diagnosis. Any change in a patient's physiological signal was quantified to assess the daily progression of the disease. After the 10th or 11th day, the possible clinical admission of patients was mentioned when the indicators resulting from the coupling of the physiological signals exceeded certain critical thresholds.

Figure 10 shows typical plots of two respiratory cases, identified with respect to the fundamental frequency component around $300 \mathrm{~Hz}$, reflecting the dynamic mechanism of standard respiratory motion. 


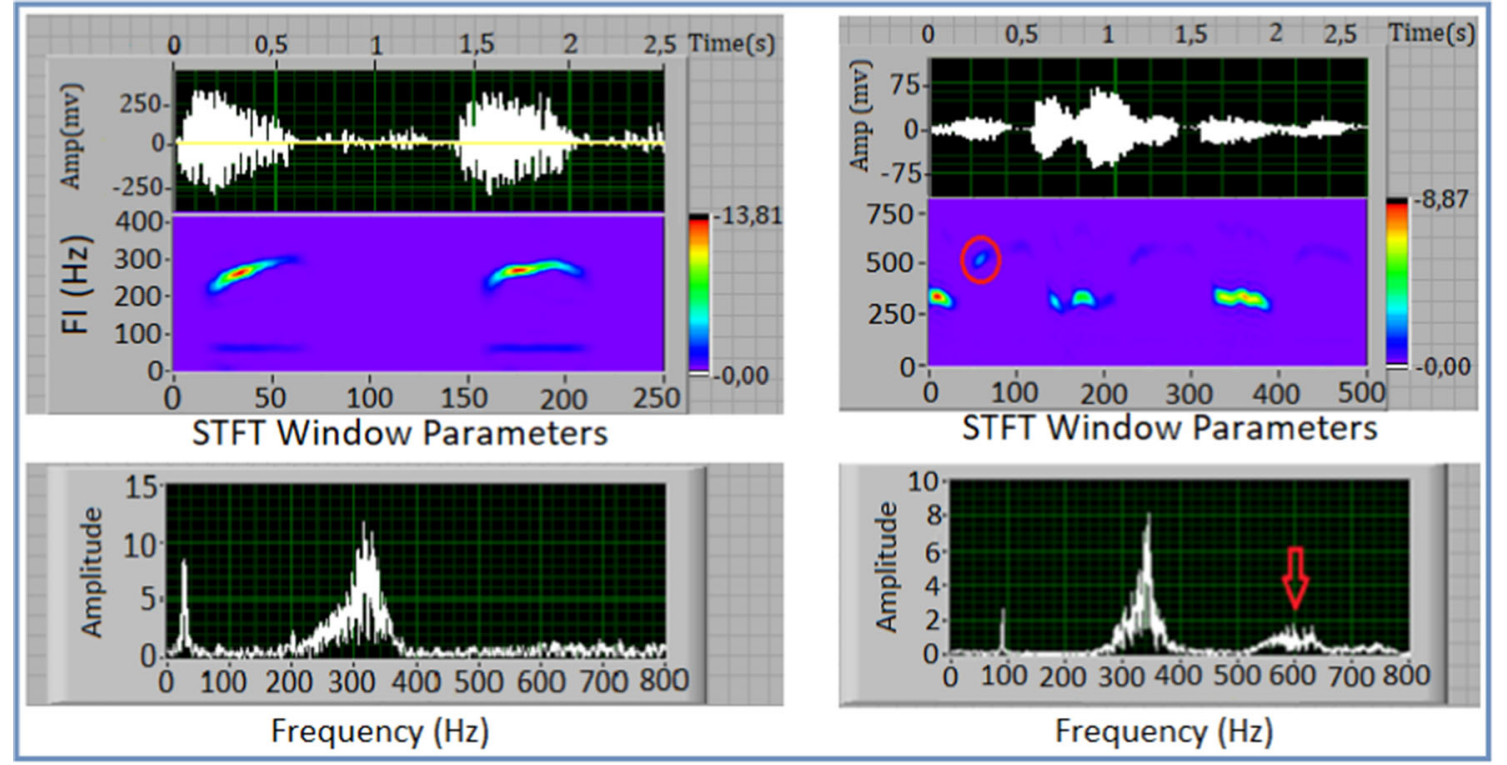

FIGURE 10. A typical illustration of the respiratory mechanism for a reference subject (left) with a regular diagram and an affected subject (right) at D4 with the presence of the wheezing frequency at $600 \mathrm{~Hz}$. On this graph, we have presented the time signal in the same frame as the STFT in order to associate the morphology of the temporal plot with its frequency spectrum.

1. Left: Standard (Healthy) case showing a regular respiratory cycle,

2. Right: Respiratory signal associated with a fever of around $38.15{ }^{\circ} \mathrm{C}(37.8-38.5)$ collected on the third day after a positive PCR test of a patient with noticeable Covid-19 symptoms. In addition to the modification that affects the vocal frequency balance (extinction of higher order frequency indices), the results highlight the appearance of a characteristic frequency around $600 \mathrm{~Hz}$ (marker) identified as an indicator of respiratory distress. This is marked by wheezing affecting the respiratory cycle.

Studying the respiratory behaviour of this population becomes very interesting. The analysis of the signals at D4 revealed a disordered evolution in the respiratory frequency diagram compared to a standard state (on the left in Fig. 10).

In addition to the morphological disturbance of the signal, a deep hissing sound was recorded at a frequency of around $600 \mathrm{~Hz}$. This characteristic plays a determining role in the diagnosis of the deterioration of the patient's condition. The attenuation over time of this component, more particularly associated with the evolution of the fever, provides decision support regarding the need for clinical admission of the patient (attenuation accompanied with high fever) or not (attenuation accompanied with a decrease in fever).

The results of the monitoring of the sound characteristics of 20 affected subjects ( 7 women, 13 men) are listed in Tables 2, 3, and 4. The different values show the close link between the dispersion of these parameters and the clinical severity of the subjects. From day
4, the analysis shows a notable variation in $\mathrm{n}$, an increase in the temporal disturbance (RTD), and an extinction of the higher-order frequency indices. Only the component of FI (1) persisted and, given its migration towards a lower frequency and its relatively broad width at half maximum, was used to conduct an objective analysis. As with the asymptomatic subjects, the average value of 0.53 recorded for the correlation coefficient compared to a healthy subject was considered as a starting value (D0).

To provide a detailed vision of the variation of the chosen characteristics in the "Affected" category (AfS), the values from three distinct stages (D0, D8, D14) extracted from the frequency components are presented in Table 3. Those resulting from the time domain are presented in Table 4 . The average fever $\left({ }^{\circ} \mathrm{C}\right)$ at each stage is also indicated in each table.

In these two tables, we have used different colours (values in red denote the average, green is the standard deviation, blue for the deviation period and orange for the final trend) to distinguish the subgroup diverging from the average. In total, 8 subjects ( 3 women and 5 men) developed acute symptoms judged clinically worrying, thus requiring admission for close clinical monitoring.

\section{DISCUSSION}

Although the number of bedside diagnoses is relatively low compared to the number of people with the disease, one of the characteristic aspects of sound waves is the ability to define alterations affecting the 
TABLE 2. Values of parameter " $n$ " at different stages under the effects of Covid-19.

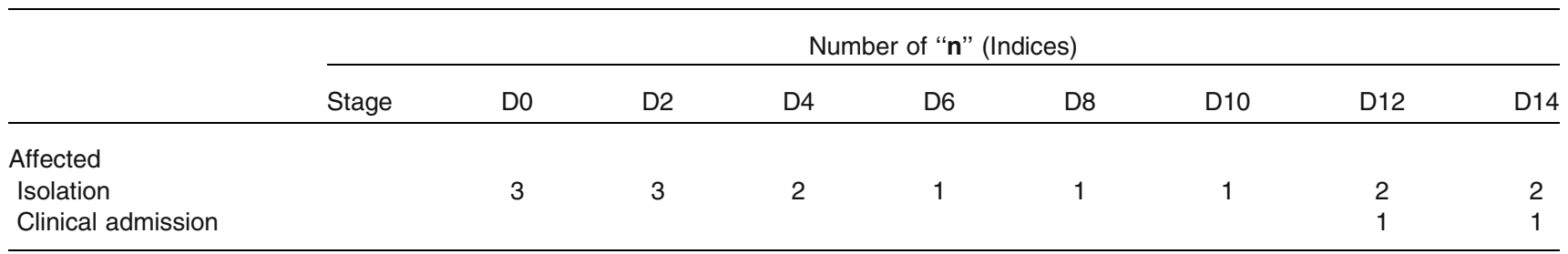

TABLE 3. Variations in frequency components.

\begin{tabular}{|c|c|c|c|c|c|c|}
\hline $\begin{array}{l}\text { Affected subject } \\
\text { (isolation) }\end{array}$ & & Indice $\left(F_{\mathrm{I}}^{(1)}\right)$ & & & $F_{\mathrm{IQ}}\left[F_{\mathrm{I}}^{(1)}\right]$ & \\
\hline Subject & D0 & D8 & D14 & D0 & D8 & D14 \\
\hline 1AfS(IS) & 47.30 & 36.38 & 39.59 & 13 & 22 & 16 \\
\hline 2AfS(IS) & 48.83 & 37.56 & 39.70 & 13 & 22 & 15 \\
\hline 3AfS(IS) & 46.75 & 35.96 & 39.31 & 14 & 23 & 17 \\
\hline 4AfS(IS) & 45.71 & 35.16 & 39.93 & 13 & 22 & 15 \\
\hline 5AfS(IS) & 48.08 & 36.99 & 40.02 & 15 & 21 & 18 \\
\hline 6AfS(IS) & 47.29 & 36.38 & 39.95 & 14 & 22 & 17 \\
\hline 7AfS(IS) & 45.52 & 35.01 & 39.89 & 15 & 21 & 18 \\
\hline 8AfS(IS) & 41.59 & 31.99 & 39.36 & 13 & 21 & 16 \\
\hline 9AfS(IS) & 42.87 & 32.98 & 40.12 & 13 & 22 & 15 \\
\hline 10AfS(IS) & 46.78 & 35.99 & 39.77 & 14 & 23 & 17 \\
\hline 11AfS(IS) & 40.86 & 31.43 & 39.91 & 12 & 21 & 14 \\
\hline 12AfS(IS) & 42.63 & 33.03 & 40.01 & 13 & 22 & 15 \\
\hline 13AfS(IS) & 44.32 & 26.99 & 25.90 & 14 & 24 & 29 \\
\hline 14AfS(IS) & 41.92 & 24.98 & 24.11 & 12 & 25 & 31 \\
\hline 15AfS(IS) & 40.63 & 26.01 & 24.79 & 13 & 24 & 31 \\
\hline 16AfS(IS) & 42.33 & 24.73 & 25.65 & 12 & 25 & 32 \\
\hline 17AfS(IS) & 41.24 & 27.99 & 26.01 & 13 & 25 & 29 \\
\hline 18AfS(IS) & 45.09 & 28.01 & 24.21 & 12 & 24 & 31 \\
\hline 19AfS(IS) & 46.09 & 26.31 & 25.11 & 14 & 26 & 33 \\
\hline 20AfS(IS) & 42.01 & 27.14 & 24.97 & 12 & 25 & 32 \\
\hline Average & 44.39 & 31.26 & 33.17 & 13.20 & 23.00 & 22.02 \\
\hline STD & 2.65 & 4.64 & 7.51 & 0.95 & 1.62 & 7.61 \\
\hline Average fever ${ }^{\circ} \mathrm{C}$ & 37.81 & $38.33 / 38.97$ & $38.21 / 38.98$ & 37.81 & $38.33 / 38.97$ & $38.21 / 38.98$ \\
\hline
\end{tabular}

vibration of the larynx during respiration. This alteration is mild to moderate for some patients and high or even severe for others.

As mentioned above, the main indicators (relative time dispersion, correlation coefficient, frequency drifts (STFT), and body temperature (fever)) were monitored and reflected the evolution of the physiological state of patients affected by marked symptoms of Covid-19.

The coupling of these indicators led to the identification and classification of three distinct pathological cases compared to a reference case (healthy subject):

(1) Asymptomatically affected

(2) Moderately affected (mild to moderate distress) with patient requiring isolation 
TABLE 4. Variations in temporal components.

\begin{tabular}{|c|c|c|c|c|c|c|c|c|c|}
\hline $\begin{array}{l}\text { Affected } \\
\text { subject } \\
\text { (isolation) }\end{array}$ & & $\mathrm{RTD}=2 \delta(1$ & & Phonic & or coef & & Respira & ory cor coef & \\
\hline Subject & D0 & $\bar{D} 8$ & D14 & D0 & $\overline{\mathrm{D} 8}$ & D14 & $\overline{\mathrm{D} 0}$ & $\overline{\mathrm{D} 8}$ & $\overline{\mathrm{D} 14}$ \\
\hline 1AfS(IS) & 1.95 & 3.83 & 3.16 & 0.57 & 0.34 & 0.44 & 0.77 & 0.37 & 0.45 \\
\hline 2AfS(IS) & 1.89 & 3.83 & 3.15 & 0.53 & 0.32 & 0.41 & 0.81 & 0.39 & 0.48 \\
\hline 3AfS(IS) & 1.97 & 4.10 & 3.18 & 0.51 & 0.31 & 0.40 & 0.83 & 0.40 & 0.49 \\
\hline 4AfS(IS) & 2.02 & 3.97 & 3.13 & 0.55 & 0.33 & 0.42 & 0.79 & 0.38 & 0.46 \\
\hline 5AfS(IS) & 1.92 & 4.00 & 3.12 & 0.53 & 0.32 & 0.41 & 0.77 & 0.37 & 0.45 \\
\hline 6AfS(IS) & 1.95 & 4.12 & 3.13 & 0.53 & 0.32 & 0.41 & 0.82 & 0.39 & 0.48 \\
\hline 7AfS(IS) & 2.03 & 3.92 & 3.13 & 0.55 & 0.33 & 0.42 & 0.79 & 0.38 & 0.46 \\
\hline 8AfS(IS) & 2.22 & 3.71 & 3.18 & 0.51 & 0.31 & 0.40 & 0.77 & 0.37 & 0.45 \\
\hline 9AfS(IS) & 2.15 & 4.22 & 3.12 & 0.57 & 0.34 & 0.44 & 0.81 & 0.39 & 0.48 \\
\hline 10AfS(IS) & 1.97 & 3.88 & 3.14 & 0.50 & 0.30 & 0.39 & 0.79 & 0.38 & 0.46 \\
\hline 11AfS(IS) & 2.26 & 3.83 & 3.13 & 0.53 & 0.32 & 0.41 & 0.78 & 0.37 & 0.46 \\
\hline 12AfS(IS) & 2.16 & 3.72 & 3.01 & 0.56 & 0.31 & 0.4 & 0.80 & 0.36 & 0.48 \\
\hline 13AfS(IS) & 2.08 & 4.26 & 4.83 & 0.51 & 0.28 & 0.24 & 0.81 & 0.30 & 0.25 \\
\hline 14AfS(IS) & 2.20 & 4.60 & 5.18 & 0.59 & 0.27 & 0.23 & 0.80 & 0.29 & 0.24 \\
\hline 15AfS(IS) & 2.27 & 4.42 & 5.04 & 0.50 & 0.27 & 0.24 & 0.82 & 0.27 & 0.23 \\
\hline 16AfS(IS) & 2.18 & 4.65 & 4.87 & 0.51 & 0.28 & 0.22 & 0.77 & 0.28 & 0.23 \\
\hline 17AfS(IS) & 2.24 & 4.11 & 4.81 & 0.49 & 0.27 & 0.23 & 0.79 & 0.28 & 0.24 \\
\hline 18AfS(IS) & 2.05 & 4.11 & 5.16 & 0.51 & 0.28 & 0.24 & 0.81 & 0.26 & 0.22 \\
\hline 19AfS(IS) & 2.00 & 4.37 & 4.98 & 0.51 & 0.28 & 0.21 & 0.80 & 0.29 & 0.24 \\
\hline $20 \mathrm{AfS}(\mathrm{IS})$ & 2.20 & 4.24 & 5.01 & 0.50 & 0.27 & 0.21 & 0.79 & 0.28 & 0.24 \\
\hline Average & 2.08 & 4.12 & 3.97 & 0.53 & 0.30 & 0.33 & 0.80 & 0.33 & 0.36 \\
\hline STD & 0.12 & 0.26 & 0.94 & 0.03 & 0.02 & 0.09 & 0.02 & 0.05 & 0.12 \\
\hline Average & & $38.33 / 38.9$ & $38.21 / 38.9$ & & $38.33 / 38.9$ & $38.21 / 38.9$ & & & $38.21 / 38.9$ \\
\hline fever ${ }^{\circ} \mathrm{C}$ & 37.81 & 7 & 8 & 37.81 & 7 & 8 & 37.81 & $38.33 / 38.97$ & 8 \\
\hline
\end{tabular}

The colour blue is attributed to subjects in subgroup 2 (affected subjects with "severe effects) for whom the characteristics at (D8) tend towards a critical state, which is confirmed at D14 (orange colour) with a divergence from the initial group average.

(3) Severely affected with acute respiratory failure and patient requiring clinical admission

Figure 11 shows the average and standard deviations of the relative variations, with respect to the reference values, of the different features able to identify each of the three categories (Healthy Patients, Asymptomatic Patients, and Affected Patients). It also demonstrates the effects of Covid-19 on the stability of the mechanism of the phonic and respiratory apparatus.

These curves show that the analysis of the characteristics extracted reflects the evolution of the effects of Covid-19 in all states. The affection of the phonic and respiratory systems leads to a migration of the voice towards the obtuse band followed by a respiratory disorder. This phenomenon can be distinguished by:

1. The decrease in the number of indices $n$, from 5 to 1 in the case of increased severity, which is why our analysis focused mainly on the index of order 1 $\left(F_{\mathrm{I}}^{(1)}\right)$.

2. The migration of the $\boldsymbol{F}_{\mathbf{I}}^{(\mathbf{1})}$ frequency indices towards lower frequencies with an increase in $\Delta \boldsymbol{F}$ in relation to the starting frequencies and a notable broadening of the index $\left(\boldsymbol{F}_{\mathbf{I Q}}\right)$ at Half-Maximum, thus reflecting a disorder of the vibratory mechanism.

3. The proportionality between the temporal fluctuation of RTD and the progression of the effects towards an acute or even worrying level.

4. The clear evolution in the correlation coefficients (phonic or respiratory) making the morphology of the signal an indicator of a deterioration in health marked by a decrease compared to the reference value, or on the contrary, recovery.

Coupled with the evolution of both body temperature and the standard deviations of each category, these different characteristics are very selective and 

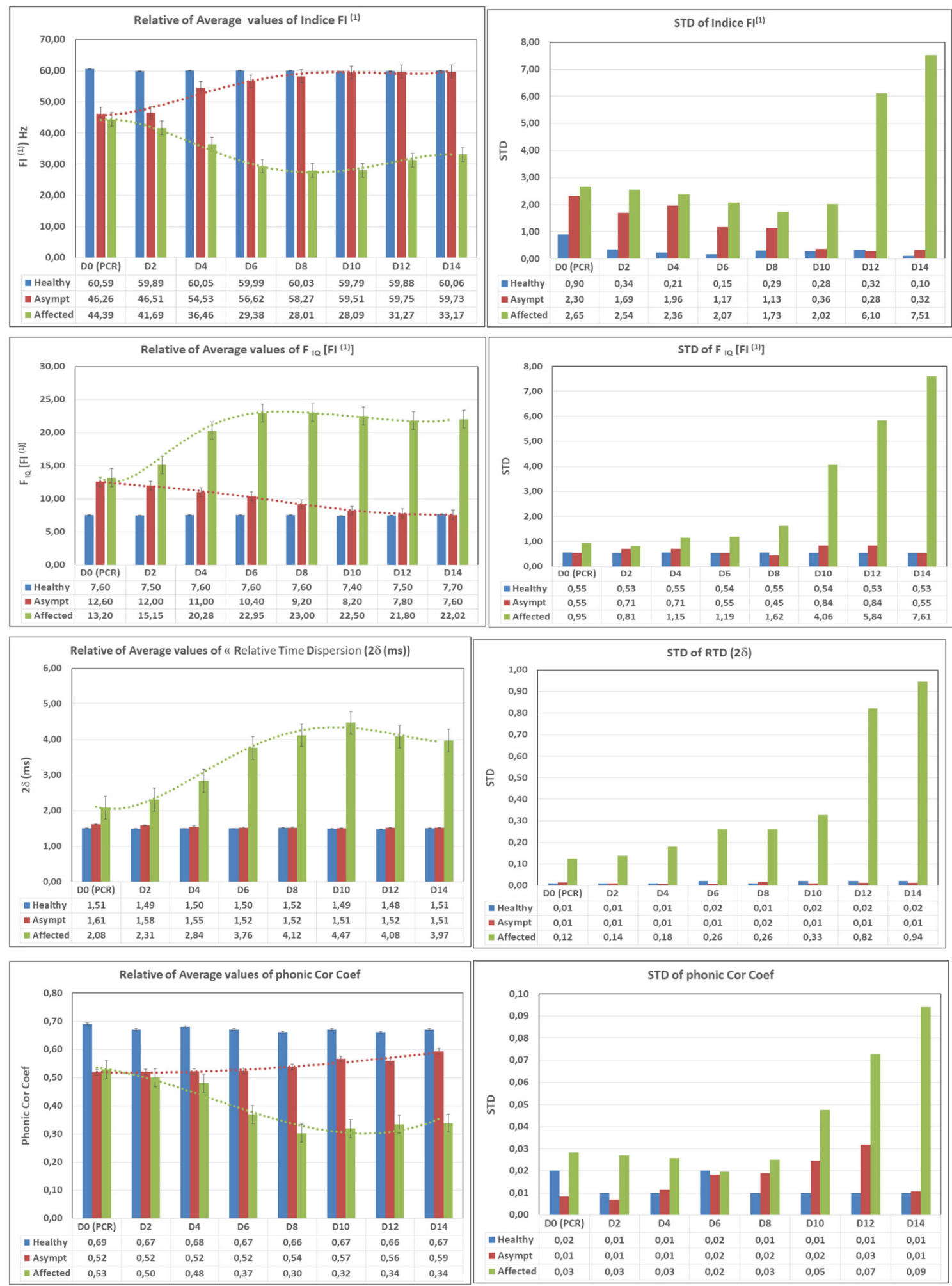

FIGURE 11. Curves showing comparative representations of the means of the different characteristics (on the left) for the three categories studied (Healthy, Asymptomatic and Affected) with their standard deviations (on the right). For the healthy and asymptomatic category, the evolutions show the viability of the features around the mean. For the affected category, the evolutions show high sensitivity and a potential to define diverging subgroups (path to recovery and need for clinical admission). 

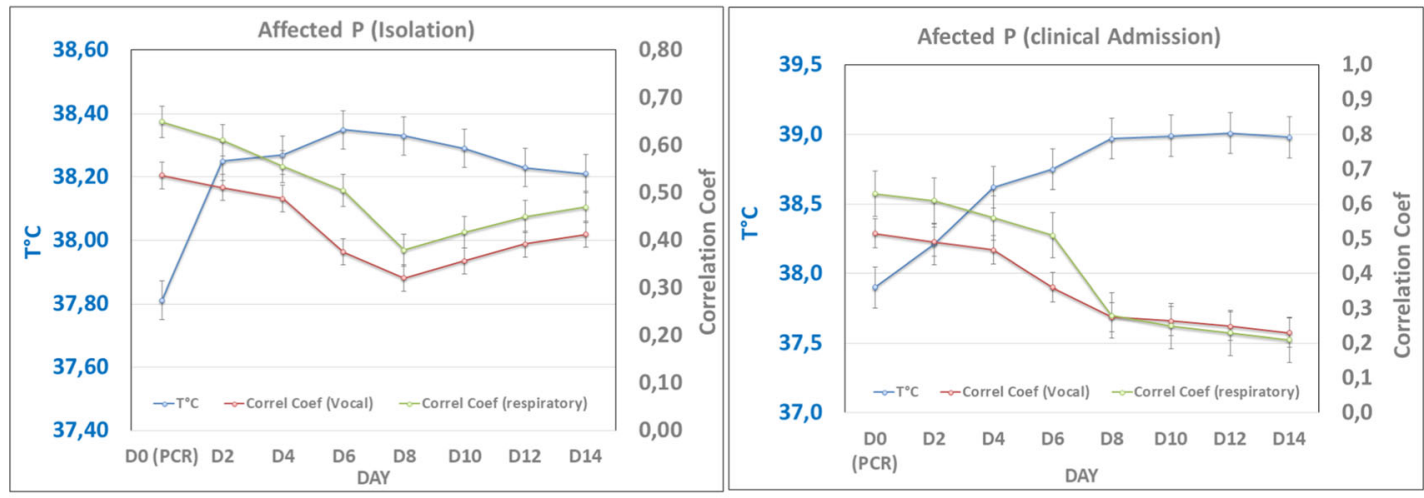

(a)
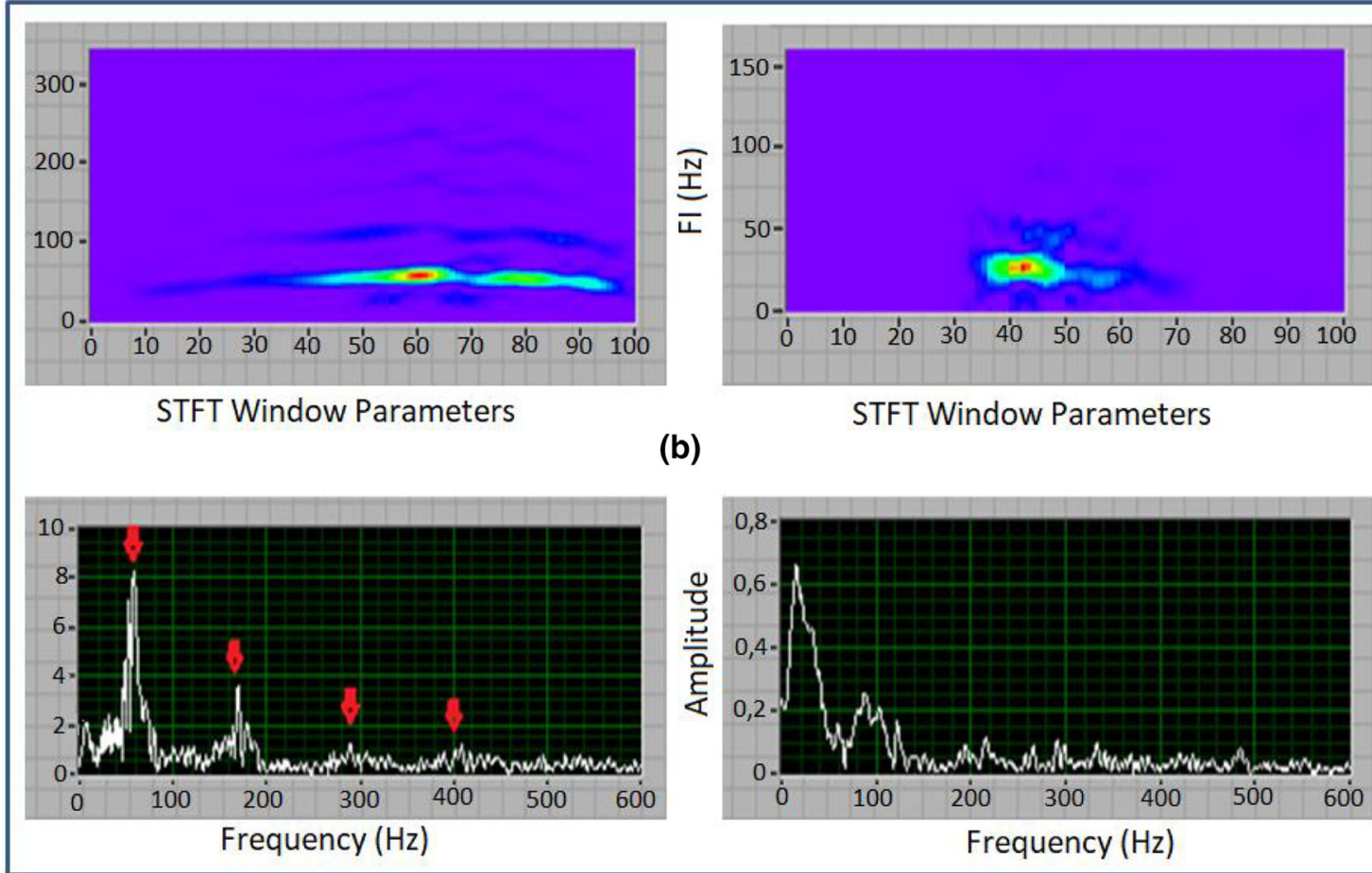

(b)

STFT Window Parameters

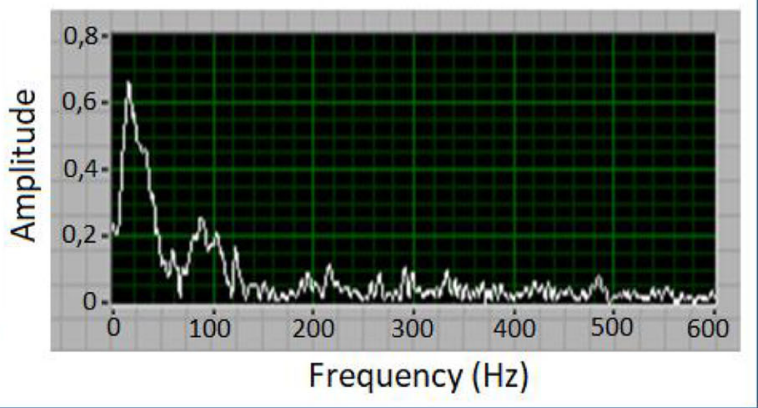

FIGURE 12. (a) Indicators of a patient affected with Covid-19 and acute respiratory distress after the 8th day: isolated (left) and requiring clinical admission (right); (b) the diagram on the left shows a return to a normal physiological state confirmed by the reappearance of the high-frequency components on the corresponding spectrum. This translates a recovery of the vocal vibration from the 8th day. This concerned 12 of the 20 subjects in the "affected" category, while the diagram on the right reflects the condition of the remaining eight patients who developed severe symptoms.

discriminatory. Indeed, their variations for the "Affected" category (subjects with acute and/or severe effects) result in a notable increase in the standard deviation thus confirming a divergence in the evolution of the states of the two subgroups forming this category.

Figure 12 shows that the third group, comprising 20 patients with acute respiratory distress, could be split into two subgroups based on the physiological indicators considered at the end of the first week:

(1) Subgroup I: the change in the slopes of the indicators suggests that the physiological state is improving and the patient is on the path to recovery.
(2) Subgroup II: the indicators continue to converge towards a critical condition that requires clinical admission for personalized care.Insofar as the condition of an affected subject evolves towards recovery, these features translate this evolution through a change in the slope and a tendency towards a value close to the average taken from the "healthy subjects" category. Conversely, the frequency and the vocal and respiratory balance of the second subgroup show a lack of coherence with the thermal indicator, which remains relatively high at $38.6{ }^{\circ} \mathrm{C}$ on average. The results indicate the need for the patient's clinical admission.As men- 


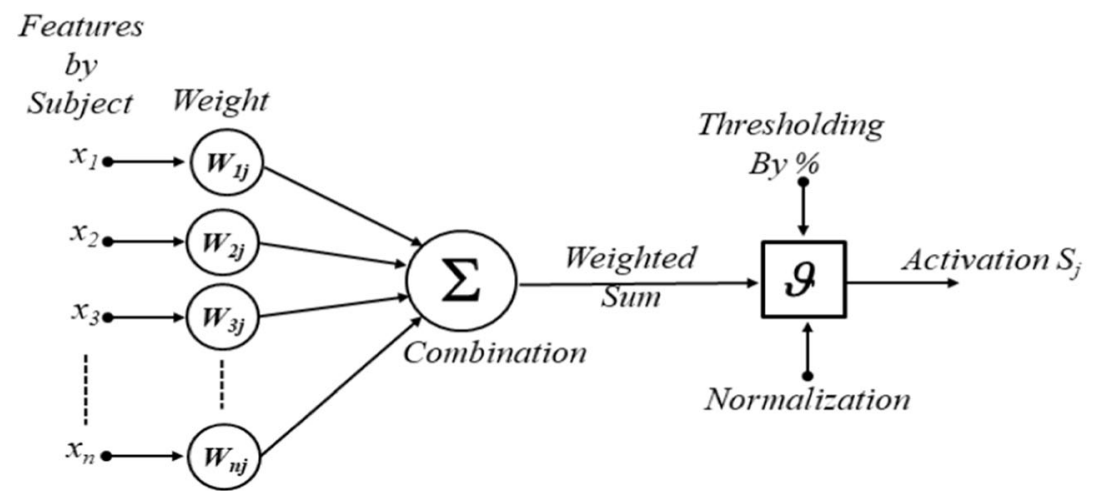

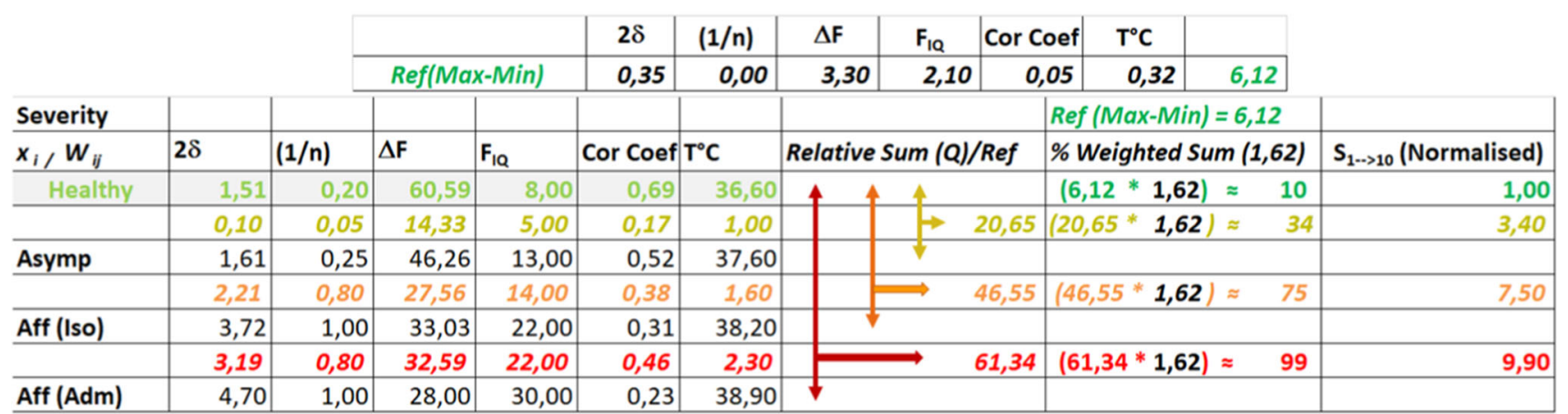

FIGURE 13. Illustration showing the basic Artificial Neural Network (ANN) architecture and an example (with contingency table) of the procedure and classification steps.

tioned above, the STFT of the respiratory signal is characterized by the appearance from D4 of a peak at $600 \mathrm{~Hz}$ associated with a whistling sound during inhalation. Continuous monitoring showed that the whistling frequency gradually disappeared and two distinct states were observed:

(3) Reappearance of the frequency components of a normal respiratory cycle (Affected P (Isolation)): the patient is recovering.

(4) Frequency inconsistency reflecting advanced illness (Affected P (Clinical admission)): the patient has to be clinically admitted.

Each characteristic can be a good indicator to differentiate the three categories. However, combining the characteristics would make it possible to classify the categories (or even the subjects) according to the severity of the effects (Fig. 13) using the basic layer of an Artificial Neural Network (ANN) concept. This individual discriminatory scale would enable the severity of each subject to be identified and classified on a standardized scale. First it was necessary to define the maximum dispersion for each characteristic in a healthy subject $(\operatorname{Ref}(|\operatorname{Max}-\operatorname{Min}|)=6.12)$ to determine the weighting coefficient (1.62). The latter was then multiplied by the sum of the differences in the char- acteristics to normalize the data to 1 . The following steps summarize the subsequent operations:

1. Subtraction of the values (designated by arrows in the table) of a random subject by category from those assigned to a "Healthy" subject.

2. Summation of the differences in all the characteristics.

3. Multiplication of the summation by the weighting coefficient.

4. Standardization on a scale of 1 to 10 .

After computing all the indicators, the patient is classified into the appropriate group according to the severity value $(S)$ computed. Figure 14 shows the classification of patients into four groups. There is a clear distinction between the asymptomatic group, the reference group (Healthy Patient), and the affected individuals that developed acute distress with a recommendation for their clinical admission, i.e., the "Affected P (Adm)" group.

The overlap in the data corresponding to the categories of the "Affected" group can be attributed to the gender and diversity of the physiological characteristics of the patients. Nevertheless, according to the results, the overlap in no way affects the estimation of the clinical severity of the subject in question. 

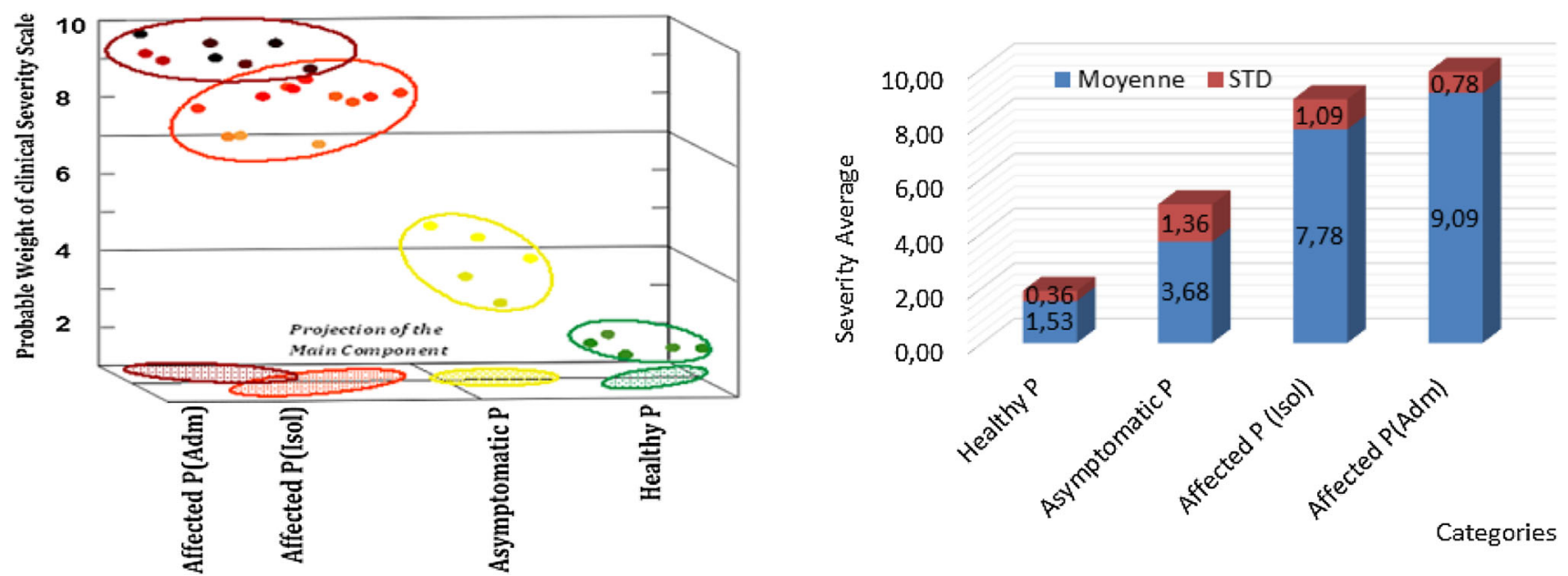

FIGURE 14. Plots showing the classification of patients into healthy patients and patients with Covid-19. The classification is based on the thermal, temporal, and frequency variations as defined by the clinical severity (S):1 (low level) to 10 (high level).

TABLE 5. (“++" High severity, “--” Low severity).

\begin{tabular}{|c|c|c|c|}
\hline Q & Cor & $\mathrm{T}^{\circ} \mathrm{C}$ & Severity \\
\hline$\longleftrightarrow$ & $\longleftrightarrow$ & $\longleftrightarrow$ & $\longleftrightarrow$ \\
\hline $\bar{\gamma}$ & 4 & $\bar{\gamma}$ & ++ \\
\hline$\pi$ & $\longleftrightarrow$ & $\pi$ & ++ \\
\hline$\longleftrightarrow$ & $\bar{\lambda}$ & 4 & +- \\
\hline $\bar{y}$ & $\pi$ & $y$ & -- \\
\hline
\end{tabular}

$\longleftrightarrow$ Constant factor
$>$ Increasing factor
4 Decreasing factor
In addition, the decision regarding the need for clinical hospitalization was taken based on the consistency between the opinions of the health professionals and the key physiological indicators given by the collar. These include more particularly an increasing or stable but high fever $\left(39^{\circ} \mathrm{C}\right)$, extinction of the high-frequency indices, and the tendency of the correlation coefficients towards zero indicating a continuous evolution of the morphology compared to the original signal. Conversely, a decrease in fever followed by stabilisation, or a positive increase in the correlation coefficients, was the consequence of a change in the morphology of the signals indicating an evolution towards the initial state, thus leading to the conclusion of a possible recovery. Consequently, the patient's condition can be qualitatively assessed and the pathophysiological evolution of the subject's severity can be predicted by combining the physiological factors (Table 5).

\section{CONCLUSION}

In a healthcare context resulting from a global respiratory pandemic, rapid and remote diagnosis may play a key role in the clinical management of patients following the onset of a pulmonary respiratory pathology associated with Covid-19. This was the focus of this study in which a passive device based on a collar was developed and implemented. The latter is equipped with two types of receptors: thermal semiconductors and ultrasound-based flexible electroactive nanowire fibres. The wearable device also comprises a central unit whose role is to ensure scheduling, signal acquisition, and remote transmission of data via a TCP/IP (USB) protocol or a Smartphone application (under development).

In addition, the in-depth understanding and quantification of biological wave/tissue interactions coupled with a data processing algorithm have led to the 
identification of key indicators that can show the pathophysiological evolution of affected patients.

The device can differentiate between patients with acute signs of respiratory failure and patients with mild symptoms and normal respiratory function. This passive listening technique has significant clinical potential for the non-invasive assessment and dynamic observation of lesions. Consequently, it merits further examination to provide support for medical operators to achieve better clinical management.

\section{SUPPLEMENTARY INFORMATION}

The online version contains supplementary material available at https://doi.org/10.1007/s12195-021-00712w.

\section{ACKNOWLEDGMENTS}

The authors would like to sincerely thank both the patients who agreed to participate despite the physical and psychological constraints and the general practitioners for their professional and personal support.

\section{ETHICAL APPROVAL}

All procedures followed were in accordance with the ethical standards of the committee in charge of human experimentation (institutional and national) and with the Helsinki Declaration of 1975, as amended in 2000 (5). Informed consent was obtained from all the patients for being included in the study.

\section{AUTHOR CONTRIBUTIONS}

All named authors have read the manuscript and have agreed to submit the paper to the Journal of Cellular and Molecular Bioengineering in its present form. The research has not been and will not be submitted simultaneously to another journal, in whole or in part. The paper reports previously unpublished work. All those named as authors have made a sufficient contribution to the work. All the authors have obtained the necessary consent from their employers or funding bodies.

\section{CONFLICT OF INTEREST}

Dany Ishac, Sandrine Matta, Sun Bin, Hussein Aziz, Elie Karam, Antoine Abche and Georges Nassar declare that they have no conflict of interest.

\section{REFERENCES}

${ }^{1}$ Ai, T., Z. Yang, H. Hou, C. Zhan, C. Chen, W. Lv, Q. Tao, Z. Sun, and L. Xia. Correlation of chest CT and RTPCR testing in coronavirus disease 2019 (Covid-19) in China: a report of 1014 cases. Radiology. 296(2):32-40, 2020. https://doi.org/10.1148/radiol.2020200642.

${ }^{2}$ Alireza, T., and A. Ardebili. Real-time RT-PCR in COVID-19 detection: issues affecting the results. Expert Rev. Mol. Diagn. 20(5):453-454, 2020. https://doi.org/10.1080/ 14737159.2020 .1757437

${ }^{3}$ Berce, V., M. Tomazin, M. Gorenjak, T. Berce, and B. Lovrenčič. The usefulness of lung ultrasound for the aetiological diagnosis of community-acquired pneumonia in children. Sci. Rep. 9(1):e17957, 2019. https://doi.org/10.10 38/s41598-019-54499-y.

${ }^{4}$ Biraja, G., and A. Tucker. Estimating uncertainty and interpretability in deep learning for coronavirus (COVID19) detection, 2020. https://arxiv.org/abs/2003.10769.

${ }^{5}$ Blundell, C., J. Cornebise, K. Kavukcuoglu, and D. Wierstra. Weight uncertainty in neural networks. In Proceedings of the 32nd International Conference on Machine Learning. Proc. Mach. Learn. Res. 37:1613-1622, 2015.

${ }^{6}$ Bouhemad, B., M. Zhang, Q. Lu, and J. J. Rouby. Clinical review: bedside lung ultrasound in critical care practice. Crit. Care. 11(1):1-9, 2007. https://doi.org/10.1186/cc5668. ${ }^{7}$ Bowen, J., Z. Ge, L. Wu, S. Wang, and M. Wan. Visualizing the mechanical wave of vocal fold tissue during phonation using electroglottogram-triggered ultrasonography. J Acoust Soc Am. 143(5):425, 2018. https://doi.org/ $10.1121 / 1.5039726$.

${ }^{8}$ Brunese, L., F. Mercaldo, A. Reginelli, and A. Santoned. Explainable deep learning for pulmonary disease and coronavirus COVID-19 detection from X-rays. Comput. Methods Prog. Biomed. 196(105608):2020, 2020. https://d oi.org/10.1016/j.cmpb.2020.105608.

${ }^{9}$ Buddhisha, U., et al. Diagnosing COVID-19: the disease and tools for detection. ACS Nano. 14(4):3822-3835, 2020. https://doi.org/10.1021/acsnano.0c02624.

${ }^{10}$ Chavez, M. A., et al. Lung ultrasound for the diagnosis of pneumonia in adults: a systematic review and meta-analysis. Respir Res. 15(1):15-50, 2014. https://doi.org/10.1186/ 1465-9921-15-50.

${ }^{11}$ Chavez, S., B. Long, A. Koyfman, and S. Y. Liang. Coronavirus disease (COVID-19): a primer for emergency physicians. American Journal of Emergency Medicine. 2020. https://doi.org/10.1016/j.ajem.2020.03.036.

${ }^{12}$ COVID-19 situation update worldwide, as of week 18, updated 12 May 2021. European Centre for Disease Prevention and Control. https://www.ecdc.europa.eu/en/ge ographical-distribution-2019-ncov-cases.

${ }^{13}$ Depeweg, S., J.M. Hernández-Lobato, F. Doshi-Velez, and S. Udluft. Decomposition of uncertainty in Bayesian deep learning for efficient and risk-sensitive learning, 2018. h ttps://arxiv.org/abs/1710.07283.

${ }^{14}$ Di, D., Z. Tang, S. Wang, H. Hui, L. Gong, Y. Lu, Z. Xue, H. Liao, F. Chen, F. Yang, R. Jin, K. Wang, Z. Liu, J. Wei, W. Mu, H. Zhang, J. Jiang, J. Tian, and H. Li. The role of imaging in the detection and management of COVID-19: a review. IEEE Rev. Biomed. Eng. 2020. https://doi.org/10. 1109/RBME.2020.2990959.

${ }^{15}$ Diab, D., et al. An autonomous low-power management system for energy harvesting from a miniaturized spherical piezoelectric transducer. Rev. Sci. Instrum. 90:e075004, 2019. https://doi.org/10.1063/1.5084267. 
${ }^{16}$ Douedi, S., and J. Miskoff. Novel coronavirus 2019 (COVID-19): a case report and review of treatments. Medicine. 99(19):e20207, 2020. https://doi.org/10.1097/M D.0000000000020207.

${ }^{17} \mathrm{Fan}, \mathrm{W}$., et al. A new coronavirus associated with human respiratory disease in China. Nature. 579:265-269, 2020. h ttps://doi.org/10.1038/s41586-020-2008-3.

${ }^{18}$ Fang, Y., H. Zhang, J. Xie, M. Lin, L. Ying, P. Pang, and W. Ji. Sensitivity of chest CT for covid-19: comparison to RT-PCR. Radiology. 296(2):200432, 2020. https://doi.org/ 10.1148/radiol.2020200432.

${ }^{19}$ Fehling, M. K., F. Grosch, M. E. Schuster, B. Schick, and J. Lohscheller. Fully automatic segmentation of glottis and vocal folds in endoscopic laryngeal high-speed videos using a deep convolutional LSTM network. PLoS ONE. 15(2):e0227791, 2020. https://doi.org/10.1371/journal.pone. 0227791

${ }^{20}$ Fukada, E., and T. Furukawa. Piezoelectricity and ferroelectricity in polyvinylidene fluoride. Ultrasonics. 19(1):3139, 1981. https://doi.org/10.1016/0041-624X(81)90030-5.

${ }^{21} \mathrm{Ha}$, U. C., et al. Binodal, wireless epidermal electronic systems with in-sensor analytics for neonatal intensive care. Science. 363:947, 2019. https://doi.org/10.1126/science.aau 0780 .

${ }^{22}$ Hamza Cherif, F., et al. Monitoring driver health status in real time. Rev. Sci. Instrum. 91(3):035110, 2020. https://doi. org/10.1063/1.5098308.

${ }^{23}$ Hayati, M., M. R. A. Bhuiyan, F. Korkmaz, and M. Nil. A review on bismuth telluride $\left(\mathrm{Bi}_{2} \mathrm{Te}_{3}\right)$ nanostructure for thermoelectric applications. Renew. Sustain. Energy Rev. 82(3):4159-4169, 2018. https://doi.org/10.1016/j.rser.2017. 10.112 .

${ }^{24}$ Kim, G.-H., S.-G. Wang, B.-J. Lee, H.-J. Park, Y.-C. Kim, H.-S. Kim, K.-T. Sohn, and S.-B. Kwon. Real-time dual visualization of two different modalities for the evaluation of vocal fold vibration - laryngeal videoendoscopy and 2D scanning videokymography: preliminary report. Auris $\mathrm{Na}$ sus Larynx. 44(2):174-181, 2017. https://doi.org/10.1016/j.a n1.2016.06.008.

${ }^{25}$ Kim, H. B., J. Jeon, Y. J. Han, Y. H. Joo, J. Lee, S. Lee, and S. Im. Convolutional neural network classifies pathological voice change in laryngeal cancer with high accuracy. J. Clin. Med. 9(11):3415, 2020. https://doi.org/10.3390/jc $\mathrm{m} 9113415$.

${ }^{26}$ Ishac, D., et al. A text-dependent speaker-recognition system. IEEE Int. Instrum. Meas. Technol. 2017. https://doi. org/10.1109/I2MTC.2017.7969677.

${ }^{27}$ Ishac, D., et al. Speaker identification based on vocal cords vibrations signal: effect of the window. Int. J. Digit. Inf. Wirel. Commun. 8(1):58-62, 2018. https://doi.org/10.17781/ P002406.

${ }^{28} \mathrm{Li}, \mathrm{X} ., \mathrm{T}$. Gu, and B. Wei. Dynamic and galvanic stability of stretchable supercapacitors. Nano Lett. 12:6366-6371, 2012. https://doi.org/10.1021/nl303631e.

${ }^{29}$ Lichtenstein, D. A. Lung ultrasound in the critically ill. Ann. Intens. Care. 4(1):1, 2014. https://doi.org/10.1186/21 10-5820-4-1.

${ }^{30}$ Majid, N., Z. Cömert, and K. Polat. A novel medical diagnosis model for COVID-19 infection detection based on deep features and Bayesian optimization. Appl. Soft Comput. 97(A):e106580, 2020. https://doi.org/10.1016/j.as oc. 2020.106580 .

${ }^{31}$ Matta, S. Acoustic wave propagation in a multilayer composed of fluid, solid, and porous viscoelastic layers.
Ph.D. Dissertation, INSA (HdF), Valenciennes (HdF), 2018. http://www.theses.fr/2018VALE0035.

${ }^{32}$ Matta, S., et al. Passive wideband concentric rings resonator for vocal cords abnormalities detection: application on larynx cancer pathologies. Rev. Sci. Instrum. 91(1):015115, 2020. https://doi.org/10.1063/1.5090193.

${ }^{33}$ Miller, S., C. Chiu, K. G. Rodino, and M. B. Miller. Pointcounterpoint: should we be performing metagenomic next generation sequencing for infectious disease diagnosis in the clinical laboratory? J Clin Microbiol. 58(3):e01739e1819, 2020. https://doi.org/10.1128/JCM.01739-19.

${ }^{34}$ Nakamura, K., and Y. Wada. Piezoelectricity, pyroelectricity, and the electrostriction constant of poly(vinylidene fluoride). J. Polym. Sci. 1971. https://doi.org/10.1002/pol. 1971.160090111.

${ }^{35}$ Nemmich, A., et al. Development of a platform dedicated to the telemedical practice under local architecture USBHID and distant TCP-IP. Int. J. Eng. Technol. 15(3):20-27, 2015.

${ }^{36}$ Neri, E., V. Miele, F. Coppola, and R. Grassi. Use of CT and artificial intelligence in suspected or COVID-19 positive patients: statement of the Italian Society of Medical and Interventional Radiology. La Radiol. Medica. 125:505508, 2020. https://doi.org/10.1007/s11547-020-01197-9.

${ }^{37}$ Papa, G. F. S., et al. Point-of-care lung sonography: an audit of 1150 examinations. J. Ultrasound Med. 36(8):16871692, 2017. https://doi.org/10.7863/ultra.16.09007.

${ }^{38}$ Peng, Q.-Y., X.-T. Wang, and L.-N. Zhang. Findings of lung ultrasonography of novel corona virus pneumonia during the 2019-2020 epidemic. Intens. Care Med. 46(5):849-850, 2020. https://doi.org/10.1007/s00134-020-0 5996-6.

${ }^{39}$ DeJonckere, Philippe H., and Jean Lebacq. In vivo quantification of the intraglottal pressure: modal phonation and voice onset. J. Voice. 34(4):645.e19-645.e39, 2020. https://d oi.org/10.1016/j.jvoice.2019.01.001.

${ }^{40}$ Principi, N., A. Esposito, C. Giannitto, and S. Esposito. Lung ultrasonography to diagnose community-acquired pneumonia in children. BMC Pulm. Med. 17(1):212, 2017. https://doi.org/10.1186/s12890-017-0561-9.

${ }^{41}$ Qian, X., et al. Current ultrasound technologies and instrumentation in the assessment and monitoring of COVID-19 positive patients. IEEE Trans. Ultrason. Ferroelectr. Freq. Control. 67(11):2230-2240, 2020. https://doi. org/10.1109/TUFFC.2020.3020055.

${ }^{42}$ Riccarda, A. Developments in biosensors for $\mathrm{CoV}$ detection and future trends. Biosensors Bioelectron. 173:e112777, 2021. https://doi.org/10.1016/j.bios.2020.112777.

${ }^{43}$ Sanaz, K., A. Mahnan, I.-L. Yeh, J. E. Aman, P. J. Watson, Y. Zhang, G. Goding, and J. Konczak. Laryngeal vibration as a non-invasive neuromodulation therapy for spasmodic dysphonia. Sci Rep. 9(1):17955, 2019. https://d oi.org/10.1038/s41598-019-54396-4.

${ }^{44}$ Sarker, L., M.M. Islam, T. Hannan, and Z. Ahmed Z. COVID-DenseNet: a deep learning architecture to detect COVID-19 from chest radiology images. Preprints. 202005015, 2020. https://doi.org/10.20944/preprints202005. 0151.v1.

${ }^{45}$ Sessler, G. M. Piezoelectricity in polyvinylidene fluoride. $J$. Acoust. Soc. Am. 70:e1596, 1998. https://doi.org/10.1121/1. 387225.

${ }^{46}$ Sheridan, C. Coronavirus and the race to distribute reliable diagnostics. Nat. Biotechnol. 38(4):382-384, 2020. https://d oi.org/10.1038/d41587-020-00002-2. 
${ }^{47}$ Shi, K., B. Sun, X. Huang, and P. Jiang. Synergistic effect of graphene nanosheet and $\mathrm{BaTiO}_{3}$ nanoparticles on performance enhancement of electrospun PVDF nanofiber mat for flexible piezoelectric nanogenerators. Nano Energy. 52:153-162, 2018. https://doi.org/10.1016/j.nanoen.2018.0 7.053 .

${ }^{48}$ Turkmen, H. I., and K. M. Elif. Advanced computing solutions for analysis of laryngeal disorders. Med. Biol. Eng. Comput. 57(11):2535-2552, 2019. https://doi.org/10. 1007/s11517-019-02031-9.
${ }^{49}$ Yang, D. Y., B. Lu, Y. Zhao, and X. Y. Jiang. Fabrication of aligned fibrous arrays by magnetic electrospinning. $A d v$. Mater. 19(21):3702-3706, 2017. https://doi.org/10.1002/ad ma.200700171.

Publisher's Note Springer Nature remains neutral with regard to jurisdictional claims in published maps and institutional affiliations. 